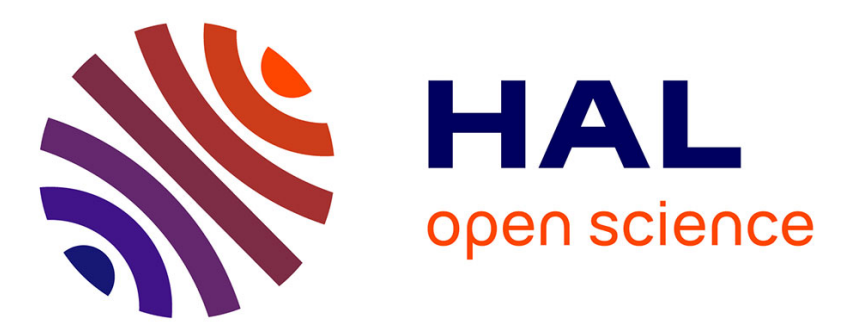

\title{
Slip localization in Inconel 718: a three-dimensional and statistical perspective
}

M.A. Charpagne, J. Hestroffer, A.T. Polonsky, M.P. Echlin, Damien Texier, V. Valle, I.J. Beyerlein, T.M. Pollock, J.C. Stinville

\section{- To cite this version:}

M.A. Charpagne, J. Hestroffer, A.T. Polonsky, M.P. Echlin, Damien Texier, et al.. Slip localization in Inconel 718: a three-dimensional and statistical perspective. Acta Materialia, 2021, 215, pp.115/117037. 10.1016/j.actamat.2021.117037 . hal-03255736

\section{HAL Id: hal-03255736 https://imt-mines-albi.hal.science/hal-03255736}

Submitted on 9 Jun 2021

HAL is a multi-disciplinary open access archive for the deposit and dissemination of scientific research documents, whether they are published or not. The documents may come from teaching and research institutions in France or abroad, or from public or private research centers.
L'archive ouverte pluridisciplinaire HAL, est destinée au dépôt et à la diffusion de documents scientifiques de niveau recherche, publiés ou non, émanant des établissements d'enseignement et de recherche français ou étrangers, des laboratoires publics ou privés. 


\title{
Slip localization in Inconel 718: a three-dimensional and statistical perspective
}

\author{
M.A. Charpagne ${ }^{\mathrm{a}, *}$, J. Hestroffer ${ }^{\mathrm{a}}$, A. T. Polonsky ${ }^{\mathrm{a}}$, M.P. Echlin ${ }^{\mathrm{a}}$, D. Texier ${ }^{\mathrm{b}}$, V. Valle ${ }^{\mathrm{c}}$, I. J. Beyerlein ${ }^{\mathrm{a}}$, T. M. \\ Pollock ${ }^{\mathrm{a}}$, J.C. Stinville \\ ${ }^{a}$ Materials Department, University of California, Santa Barbara, CA 93106, USA \\ ${ }^{b}$ Institut Clement Ader (ICA) - UMR CNRS 5312, Universit de Toulouse, CNRS, INSA, UPS, Mines Albi, ISAE-SUPAERO, Campus Jarlard, 81013 \\ Albi Cedex 09, France \\ ${ }^{c}$ Institut PPRIME, Université de Poitiers, CNRS, ENSMA, UPR 3346, 86962 Chasseneuil Cedex, France
}

\begin{abstract}
The slip localization behavior of the polycrystalline nickel base superalloy Inconel 718 during monotonic tensile loading at room temperature, is investigated for the first time in relation to the $3 \mathrm{D}$ microstructure. Multi-modal data merging tools are used to recombine high resolution digital image correlation (HR-DIC) data with 3D electron backscatter diffraction tomography (3D EBSD), over a wide region of interest. This procedure enables reconstruction of the slip band planes in the 3D microstructure. Statistical analyses conducted over 500 individual slip bands reveal strong correlations between their location and specific microstructure configurations. In particular, over half of the slip bands emanate from triple junction lines (3D lines defined by the junction of three crystals). Moreover, the most intense and longest slip bands, which would become critical fatigue crack nucleation sites during cyclic loading, are located close and parallel to particular annealing twin boundaries and are simultaneously connected to triple junction lines. Crystal plasticity finite elements calculations are performed on the experimental microstructure to identify the slip activity that results in the formation of high intensity slip bands (localized plasticity) or zones of high lattice rotation (non-localized plasticity) in these particular microstructure regions.

Keywords: Strain Localization, High Resolution Digital Image Correlation, 3D EBSD, Slip bands, Crystal Plasticity Finite Elements
\end{abstract}

\section{Introduction}

In the absence of manufacturing defects, such as inclusions and pores, fatigue crack nucleation sites in polycrystalline nickel base superalloys are strongly correlated to the grain structure [1-3]. These cracks are the result of strain localization phenomena that manifest in the form of localized subgrain defects usually referred to as persistent slip bands, fatigue shear bands or slip bands [4-8]. The microstructure locations where the first slip bands form can be identified within the first few loading cycles [8]. Therefore, the accurate mapping of incipient strain concentration

*corresponding author

Email address: mcharpagne@ucsb .edu (M.A. Charpagne) 
areas in the materials microstructure is of critical importance for the prediction of crack nucleation sites and ultimately the design of microstructures of improved fatigue resistance.

Numerous studies have focused on mapping slip localization phenomena in nickel base superalloys [9-13]. They generally involve the use of high resolution imaging such as atomic force microscopy, optical or scanning electron microscopy (SEM) or more recently high resolution digital image correlation (HR-DIC), to capture the highly localized slip events traces at the specimens free surface. The observed slip traces are the result of the glide of multiple $\frac{1}{2}\{111\}<110>$ dislocations on an octahedral plane in the face centered cubic lattice. When coupled to grain structure information, usually acquired using electron back scatter diffraction (EBSD), these investigations reveal that some annealing twin boundaries are particularly prone to trigger the formation of critically intense slip bands in their vicinity. In these face centered cubic materials, annealing twin boundaries are defined as $60^{\circ}$ rotations around $<111>$ directions. Some but not all of these intense slip bands exhibit substantial irreversibility during cyclic loading and ultimately promote crack nucleation [8]. Studies have enabled the definition of crystallographic-based criteria to predict along which twin boundaries intense bands are prone to develop [11]. However, these criteria are insufficient to predict the location and intensity of all slip events within the materials microstructure. One possible explanation is that the surface measurements used to develop these criteria do not fully render the completeness of the threedimensional (3D) grain structure, and that the slip traces visible on a specimens surface emanate from a variety of different sub-surface sources. Of potential importance are sub-surface grain neighborhoods as well as the networks of grain boundary surfaces and triple junction lines (3D lines formed by the junction of three grains).

In the present article, HR-DIC measurements acquired during monotonic loading are coupled to 3D microstructure data, to elucidate the relationship between the microstructure and the location of the most critical slip events. To do so, automated multi-modal data merging methods are developed and deployed to reconstruct the slip bands into the 3D microstructure over a large (millimetric) region of interest. These methods enable a full identification of the morphology, location and connectivity of the slip bands in three dimensions. Analytical tools are used to gain a statistical perspective of the slip bands location and intensity as a function of the microstructure features.

\section{Experimental Materials}

\subsection{Wrought Inconel 718}

Wrought Inconel 718 (nominal composition in wt $\% 0.56 \% \mathrm{Al}-17.31 \% \mathrm{Fe}-0.14 \% \mathrm{Co}-17.97 \% \mathrm{Cr}-5.4 \% \mathrm{Nb}-$ $1.00 \% \mathrm{Ti}-0.023 \% \mathrm{C}-0.0062 \% \mathrm{~N}-$ bal $\mathrm{Ni}$ ) was subjected to a 30 minutes annealing treatment at $1050{ }^{\circ} \mathrm{C}$ followed by water quenching. This treatment yields a controlled grain size distribution centered around $62 \mu \mathrm{m}$ with limited texture, as revealed by the inverse pole figure (IPF) shown in Figure 1-a. Like most FCC materials with low to medium stacking fault energy, most grains contain coherent annealing twin boundaries. A two-steps precipitation hardening treatment consisting of 8 hours exposure at $720^{\circ} \mathrm{C}$ then 8 hours at $620^{\circ} \mathrm{C}$ was conducted to form hardening $\gamma^{\prime}$ and $\gamma$ " precipitates[14], which are shown in the bright field transmission electron micrograph in Figure 1-b. The 
material did not contain any visible delta phase particles. Flat dogbone-shaped tensile specimens of gage section $0.7 \times 3 \mathrm{~mm}$ were cut by wire electrical discharge machining, mechanically mirror polished using abrasive papers and diamond suspensions, then chemo-mechanically polished using a suspension of $0.04 \mu \mathrm{m}$ colloidal silica particles before mechanical testing. The engineering stress-strain curve is shown in Figure 1-c.

a)

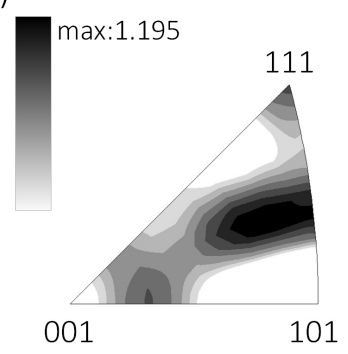

b)

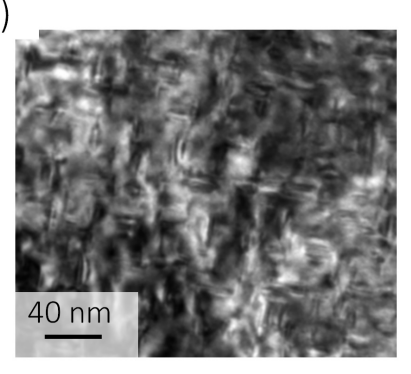

c)

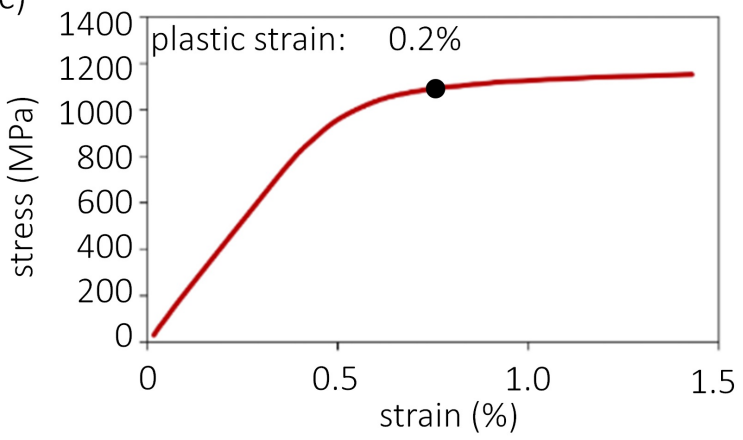

Figure 1: Structure and properties of the studied Inconel 718 nickel base superalloy: a) texture index-colored IPF plot (the projected direction is the loading direction), b) bright field transmission electron micrograph showing the $\gamma$ " hardening precipitates, c) engineering stress-strain curve.

\subsection{High Resolution Digital Image Correlation and 3D microstructure measurements}

The region of interest $(\mathrm{ROI})$ consists of a $549(\mathrm{X}) \times 420(\mathrm{Y}) \times 526(\mathrm{Z}) \mu \mathrm{m}^{3}$ volume, $\mathrm{X}$ being the loading direction. HR-DIC data was acquired on the (XY) surface and 3D EBSD over the whole volume. Both datasets were acquired using a Versa 3D Thermo Fischer Dual-Beam SEM. An overview of the datasets is shown in Figure 2. 
a)

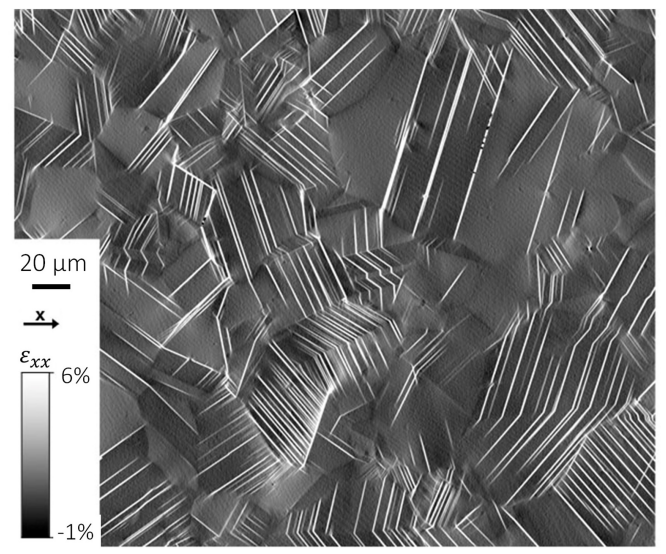

C)

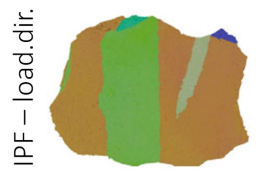

d)

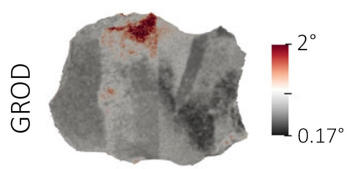

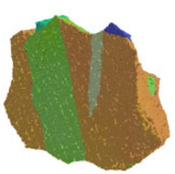

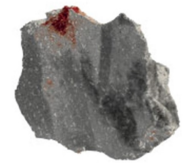

b)

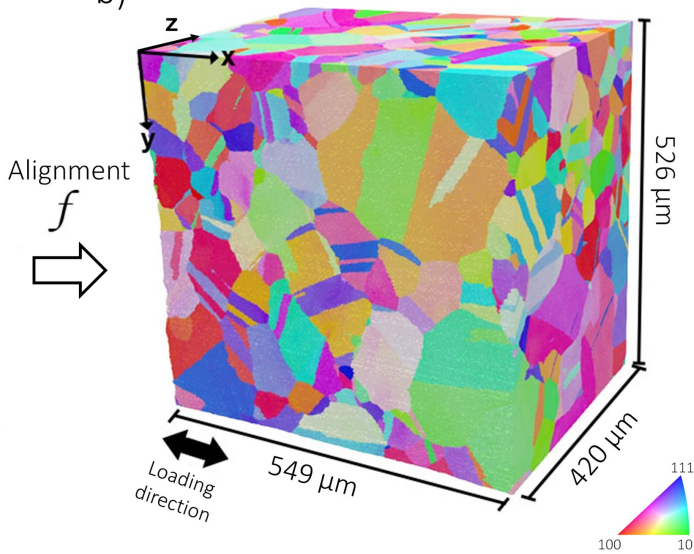

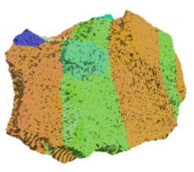
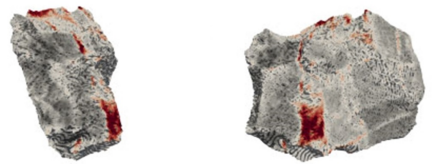

Figure 2: Collected datasets and merging workflow. a) Strain field $\epsilon_{x x}$ along the loading direction after deformation at $0.2 \%$ plastic strain; bands of concentrated strain are the locations of slip events (slip bands). b) 3D EBSD dataset collected using Tribeam tomography after deformation of the specimen at $1.83 \%$ macroscopic strain (1.26\% plastic strain). The front surface corresponds to the free surface of the specimen where HR-DIC data was collected. The color code indicates the crystallographic orientation along the loading direction. c-d) 3D representation of a grain, colored according to the crystallographic orientation along the loading direction (c) grain reference orientation deviation (d).

Prior to deformation, a speckle pattern consisting of $60 \mathrm{~nm}$ gold nanoparticles was deposited on the surface of the sample, following the procedure by Kammers et al. [15]. The specimen was strained in-situ in the SEM, using a custom built stage. The macroscopic strain was measured in-situ using fiducial markers located at both ends of the gage section. Loading was interrupted at a plastic strain of $0.2 \%$ (corresponding to the macroscopic yield) for HR-DIC data collection, and then further up to $1.83 \%$ macroscopic strain where more HR-DIC data was collected. As the present article focuses on incipient plasticity, the slip bands that developed at the macroscopic yield only are studied. Subset sizes of 31 pixels $\times 31$ pixels $(1044 \mathrm{~nm} \times 1044 \mathrm{~nm})$ and $27 \times 27$ pixels $^{2}(910 \mathrm{~nm} \times 910 \mathrm{~nm})$ with a step size of 3 pixels $(101 \mathrm{~nm})$ were used, in accordance with the number of particles per subset recommended by Sutton [16]. Sets of SEM images were acquired before loading and after unloading from plastic deformation. To minimize the distortion errors inherent to SEM, imaging parameters were chosen following the guidelines of Kammers and Daly [17, 18] and Stinville et al. [19]. High magnification images were taken at a horizontal field width of 138 $\mu \mathrm{m}$. Large electron beam spot sizes and large dwell times $(20 \mu s)$, were used to reduce drift distortions. A low acceleration voltage $(10 \mathrm{kV})$ was used to minimize charging effects and drift distortions, and to obtain higher spatial 
imaging resolution and electron beam stability. A National Instruments scan controller and acquisition system (DAQ) were used to control beam scanning in the microscope and remove the SEM beam defects associated with the scan generator [19, 20]. Image correlation was performed using the discontinuity-tolerant Heaviside-DIC method [21, 22]. This method enables high resolution mapping and quantitative measurement of the plastic deformation. The strain map obtained from HR-DIC after deformation to a plastic strain of $0.2 \%$ is displayed in Figure 2-a. In addition, the Heaviside-DIC method also provides quantitative measurements of in-plane slip with a resolution in the order of 10 $\mathrm{nm}$. The in-plane slip corresponds to the physical in-plane displacement produced by slip bands onto the specimen free surface. Furthermore, the Heaviside-DIC method enables, with knowledge of the crystallographic orientation, identification of the active slip system in each slip band [22].

The 3D microstructure dataset was acquired using Tribeam tomography $[23,24]$ after unloading from the final macroscopic strain. EBSD data was collected on a series of 526, $1 \mu \mathrm{m}$ thick, consecutive slices, using an acceleration voltage of $30 \mathrm{kV}$ and a $4 \times 4$ binning on a EDAX OIM-Hikari XM4 EBSD detector. An (XY) step size of $1 \mu \mathrm{m}$ was used on each slice to produce cubic voxels. Ablation of the material between each slice is achieved using a femtosecond laser, which produces minimal surface damage. EBSD patterns were indexed using the Dictionary Indexing method developed by DeGraef et al. [25-27]. The 3D volume was reconstructed using the Bluequartz DREAM.3D open source software [28]. Individual grains were segmented using a misorientation tolerance angle of $5^{\circ}$ and twin boundaries were defined with a $2^{\circ}$ tolerance on both the plane and direction. The Paraview [29] software was used for data visualization. The grain reference orientation deviation (GROD) parameter was calculated for each voxel. It is defined as the difference between the local crystallographic orientation and the average crystallographic orientation of the grain, as shown in Equation 1, where $o_{(x, y, z)}$ is the orientation of the voxel at the location $(x, y, z)$ and $o_{g}$ is the average orientation of the grain $g$ to which the voxel belongs. This procedure provides unique identification of the $3 \mathrm{D}$ grain clusters (twins and parent grains) as shown by the sequential images (rotation around the $\mathrm{Y}$ axis) in Figure 2-c. Since 3D microstructure data was extracted after straining, the lattice rotation produced during deformation is readily measured, as shown using the GROD metric in Figure 2-d.

$$
\operatorname{GROD}(x, y, z)=\left|o_{(x, y, z)}-o_{g}\right|
$$

\section{Reconstructing slip bands in 3D using multi-modal data vectorization and merging}

A set of pairs of control points was selected to align the DIC data onto the XY surface of the 3D volume. The distortion between both datasets was modeled using a polynomial function of degree $3, f$ (figure 2 , which formula is given in Equation 2) [30]. Individual slip traces were segmented from the DIC maps and indexed as individual features, using the iterative Hough transformation method described in [12]. The location of each slip band in the 3D volume (coordinates of its endpoints on the (XY) surface), its inclination angle relative to the loading direction, its length and average in-plane slip intensity and direction are calculated. With the assumption of slip on the $12\{111\}\langle 110\rangle$ slip 
systems, the recombination of the slip trace inclination angle with the local crystallographic orientation enables the determination of the active $\{111\}$ slip plane. As the direction of the shearing produced by the slip band on the free surface is readily measured, the Heaviside method enables the determination of the active $<110>$ slip direction as well [22]. Both the slip plane and direction are identified automatically using the procedure detailed in [12]. Since at least one point within each slip band and its crystallographic slip plane are known, all the slip bands can be projected and reconstructed in the 3D volume. An example is shown in Figure 3. Let $\vec{n}_{\text {crystal }}$ be the normal vector to the slip plane in the crystal reference frame. For a slip band which active slip plane is $\{\mathrm{hkl}\}, \vec{n}_{\text {crystal }}=(h, k, l)$. The coordinates of this normal vector in the sample reference frame, $\vec{n}_{\text {sample }}$, are obtained by rotating $\vec{n}_{c r y s t a l}$ according to equation 3 , where $\mathbf{R}_{\text {sample,crystal }}$ is the passive rotation matrix that brings the sample reference frame (XYZ) to coincide with the crystal reference frame. Then, let us name $A$ and $B$ the slip trace endpoints on the DIC surface (see Figure 3-a), in the sample reference frame. By definition, a voxel $P$ of coordinates $(x, y, z)$ belongs to the slip band if $\overrightarrow{A P} . \vec{n}_{\text {sample }}=0$ (similarly, $\overrightarrow{B P} \cdot \vec{n}_{\text {sample }}=0$ ). Owing to the voxelized nature of the data, some angular tolerance was applied to that condition in order to obtain a 1 voxel thick slip band. The voxels that fulfill this condition were assigned the slip band number ID, to create a 3D rendering. Individual slip bands are viewed either as such (voxelized objects), as shown in dark grey in Figure 4-a; or as planar objects defined by a point-normal couple $\left(A, \vec{n}_{\text {sample }}\right)$ in Paraview, as shown in Figures 3-b and 6.

a)

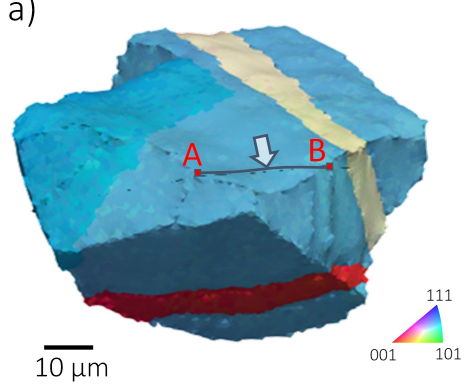

b)

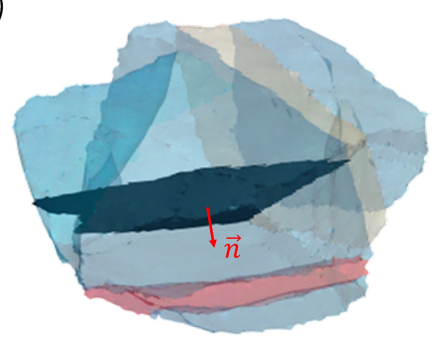

Figure 3: Reconstruction of a 3D slip band from a 2D slip trace: a) grain of interest and slip trace overlapped onto the free surface (highlighted with the arrow), A and B are the coordinates of the endpoints of the slip trace; b) 3D slip band reconstructed after multi-modal data merging and determination of the active $\{111\}$ plane (the plane normal $\vec{n}$ is highlighted with a red arrow).

$$
\begin{gathered}
f(x, y)=\left\{\begin{array}{l}
x^{\prime}=\sum_{n=0}^{3} \sum_{k=0}^{n} c_{k, n-k}^{x} x^{k} y^{n-k} \\
y^{\prime}=\sum_{n=0}^{3} \sum_{k=0}^{n} c_{k, n-k}^{y} x^{k} y^{n-k}
\end{array}\right. \\
\vec{n}_{\text {sample }}=\mathbf{R}_{\text {sample,crystal }} \vec{n}_{\text {crystal }}
\end{gathered}
$$


a)

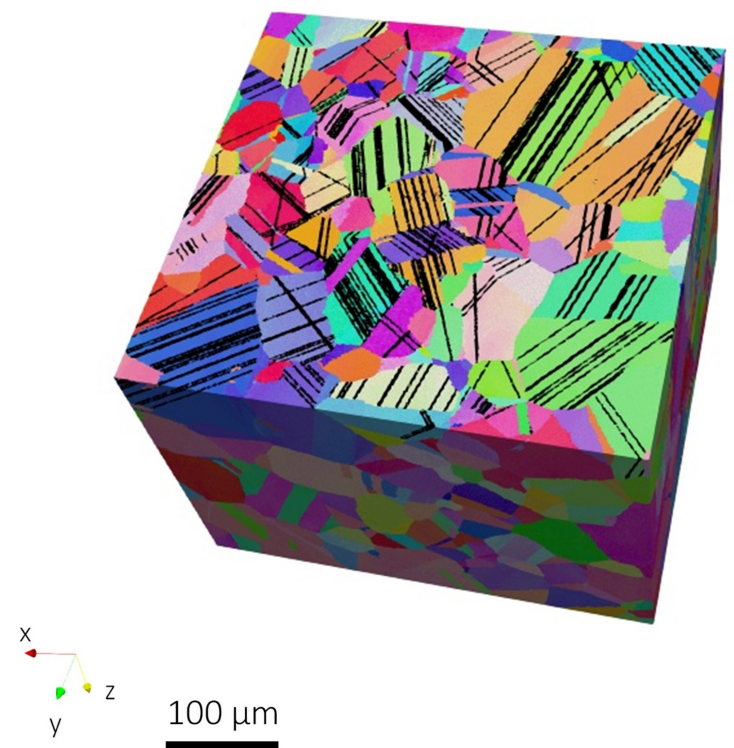

b)

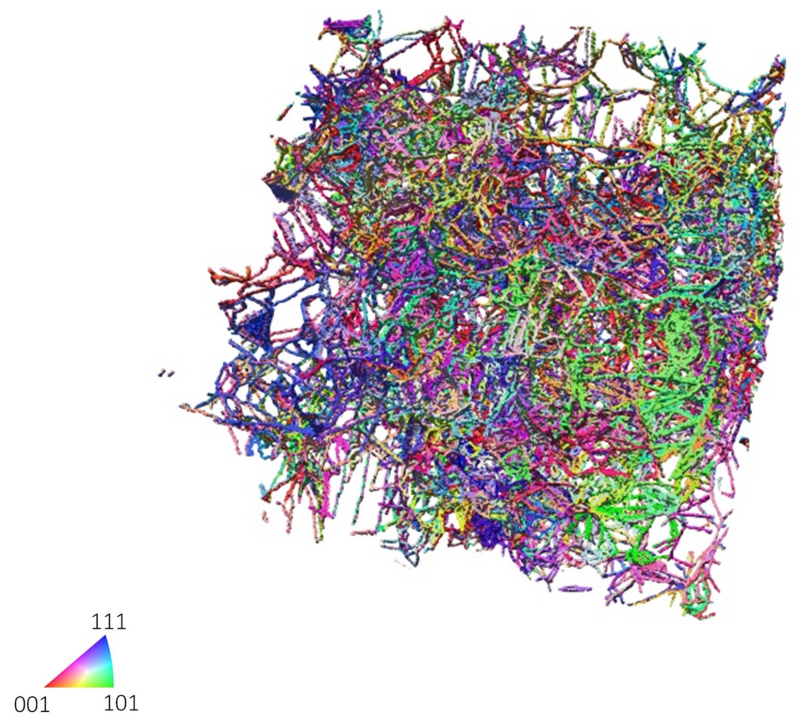

Figure 4: Merged dataset, in IPF colors according to the loading direction: a) slip bands overlapped to the 3D microstructure as black planes; b) individual triple junction lines.

As they are microstructure objects of interest, twin boundaries and triple junction lines were also segmented as individual features. Each twin boundary separates two crystals in a twin orientation relationship while each triple junction line is defined as the junction of three individual 3D crystals. A representation of the triple junction lines is shown alongside the merged dataset in Figure 4-b.

\section{Simulation Methods}

3D crystal plasticity finite element (CPFE) calculations were performed to connect the mechanical response of local clusters of grains to underlying crystallographic slip and lattice rotation. The approach includes building 3D experimentally informed microstructures authentic in their morphology and crystallographic representation of the grain boundaries, triple lines, and quadruple points, a critical step that is non-standard and hence will be detailed after a brief review of the constitutive law used.

\subsection{Crystal plasticity model}

The constitutive law is the large strain formalism for elasticity and crystallographic slip developed by Kalidindi, Bronkhorst, and Anand [31]. Minor modifications were made to incorporate strain hardening via a dislocation density based hardening law. Kinematics of the model follow closely that of Asaro [32] and relate the deformation gradient, F, and velocity gradient, $\mathbf{L}$, to elastic deformation and slip for every material point of the mesh. For the present material, the model considers plastic deformation to take place solely via dislocation slip on the $12\{111\}\langle 110\rangle$ FCC 
close-packed slip systems. The kinematics and constitutive laws defined in the model were introduced in the form of a user-defined mechanical material behavior (UMAT) subroutine implemented in ABAQUS/Standard.

In this model, the activity of a slip system, $\alpha$, is given by its slip rate, $\dot{\gamma}^{(\alpha)}$. This is related to the resolved shear stress on the system, $\tau^{(\alpha)}$, by the flow rule. This work implements a rate-dependent power-law flow rule similar to that suggested by Peirce, Rice and Needleman [33]

$$
\dot{\gamma}^{(\alpha)}=\dot{\gamma}_{0}\left|\frac{\tau^{(\alpha)}}{g^{(\alpha)}}\right|^{n} \operatorname{sgn}\left(\tau^{(\alpha)}\right)
$$

where $\dot{\gamma}_{0}$ represents the reference slip rate, $g^{(\alpha)}$ the slip resistance on system $\alpha$, and $n$ the power-law exponent. Slip resistance evolves according to a generalized tensor form of the classic Taylor relation suggested by Franciosi et al. [34] taking into account the average interaction strengths between slip systems, given in Equation 5

$$
g^{(\alpha)}=g_{0}+\chi b \mu \sqrt{\sum_{\beta=1}^{n} a^{\alpha \beta} \rho^{(\beta)}}
$$

where $g_{0}$ is the structure independent slip system strength, $\chi$ a statistical dislocation interaction coefficient equal to unity, $b$ the value of the Burgers vector, $\mu$ the shear modulus, $a^{\alpha \beta}$ a slip-interaction matrix capturing self-hardening and latent hardening coefficients, and $\rho^{(\beta)}$ the dislocation density on system $\beta$. The dislocation densities for each system evolve according to the Kocks-Mecking equation (Equation 6) [35], composed by a positive storage rate term and a negative dynamic recovery term

$$
\dot{\rho}^{(\alpha)}=\frac{1}{b}\left(\frac{1}{K} \sqrt{\sum_{\beta=1}^{n} a^{\alpha \beta} \rho^{(\beta)}}-2 y_{c} \rho^{(\alpha)}\right)\left|\dot{\gamma}^{(\alpha)}\right|
$$

where $K$ is a constant that describes dislocation junction strength, and $y_{c}$ is the critical annihilation distance. Table 1 lists the values of the constitutive model parameters. Details of the parameter selection and calibration process can be found in the Supplement.

\subsection{Voxel-to-Mesh Procedure}

Experimentally informed 3D models of the materials microstructure were created through a multi-stage workflow designed to produce analysis-ready meshes with high-fidelity microstructure representations from voxelized data. The method is general to any complexity of microstructure and size, yet in its description small target groups of grains possessing triple lines requiring analysis were targeted. Figure 5-a shows an example of one of many small groups of grains extracted from the large 3D EBSD dataset.

The approach consists of three main steps. The first step is a voxel-to-mesh procedure that transforms voxelized microstructure into a topologically refined, fully mirrored grain cluster with smooth grain boundaries, triple junction lines and quadruple points. The second one involves embedding the cluster within a coarse grain environment, including free surfaces, and the final step is application of deformation conditions. 
Table 1: Constitutive model parameters.

\begin{tabular}{ccc}
\hline Parameter & Value & Unit \\
\hline$C_{11}$ & 259.6 & $\mathrm{GPa}$ \\
$C_{12}$ & 179 & $\mathrm{GPa}$ \\
$C_{44}$ & 109.6 & $\mathrm{GPa}$ \\
$\dot{\gamma}_{0}$ & $10^{-3}$ & $\mathrm{~s}^{-1}$ \\
$n$ & 20 & - \\
$g_{0}$ & 400 & $\mathrm{MPa}$ \\
$\mathrm{b}$ & 0.257 & $\mathrm{~nm}$ \\
$\alpha^{\alpha \beta}$ & 0.1 & - \\
$\rho_{0}$ & $1.5 \times 10^{12}$ & $\mathrm{~m}^{-2}$ \\
$K$ & 10 & - \\
$y_{c}$ & 2.57 & $\mathrm{~nm}$ \\
\hline
\end{tabular}

The first step in the voxel-to-mesh workflow begins with a data preparation stage. To create the grain cluster, the dataset was cropped to a subvolume centered around a microstructure feature of interest (boundary, grain aggregate, triple junction line). These subvolumes then underwent a defeaturing process whereby non-ideal voxels are marked for grain ID reassignment based on two criteria. The first ensures a minimum allowed grain-size in the model, measured as the number of voxels belonging to a particular grain. If a voxel belongs to an exceedingly small grain (2 voxels), it is marked for reassignment. The second criterion considers the neighborhood around each voxel. Voxels with less than three face-adjacent neighbors of the same grain are marked for reassignment. Such voxel neighborhoods typically result in relatively poor quality mesh elements in the final model. After all voxels have been processed, those selected for reassignment are treated as void/unassigned material and are filled-in through an iterative grain growth process by adopting the most abundant grain ID in their neighborhood until no more unassigned voxels remain. The defeaturing step is repeated to minimize the number of non-ideal voxel neighborhoods.

The next stage involves generating smoothed surface meshes for each subvolume of microstructure. For this, the open source software Voxel2Tet is used [36]. Taking voxelated data as input, Voxel2Tet implements a volume preserving Laplacian smoothing algorithm, outputting boundary conforming surface mesh for every grain in the subvolume. The Voxel2Tet algorithm preserves all of the original vertices of its input and does not create or interpolate new ones. This results in surface meshes that hold true to the experimental data at its provided resolution. Once complete, the grain surface meshes are combined to form the overall microstructure surface mesh from which to carry out volume meshing.

Given the complexity of real polycrystalline microstructures, when creating the volumetric mesh, it is critical to create robust 3D mesh for any general surface mesh provided. Here, volumetric meshing was carried out using the 
open source software TetWild [37]. An example is given in figure 5-b. The algorithm used by TetWild computes approximately constrained tetrahedralizations from any input surface. This allows for a user-defined deviation from input surface mesh, critical for the performance of the algorithm and final mesh quality. For details of the TetWild algorithm as well as comparisons with other state of the art tetrahedralization algorithms (see Hu et al. [38]). For the microstructure models, an isotropic adaptive meshing scheme was applied. In this scheme, an ideal edge length is prescribed uniformly for the whole structure, and adaptive refinement is only carried out to preserve surface mesh topology. The resulting meshes consisted of approximately 1 million, 10-noded tetrahedral elements (C3D10) for each model. After the meshing was complete, elements were reassigned to their respective grain ID by determining which grain surface mesh they are inside of using a generalized winding number algorithm [39].

When considering the boundary conditions for the models, five of their six cubic faces are constrained due to surrounding microstructure while the one other corresponds to a free surface. The unstructured mesh topology of the models in combination with these constraints makes applying periodic boundary conditions difficult. Instead, an additional meshing operation was performed to create a homogeneous matrix material surrounding the models. Elements of the surrounding mesh located above the free surface plane of the model were modeled as isotropic elastic material with extremely low modulus, $E=1 \times 10^{-10} \mathrm{GPa}$, to mimic an unconstrained surface (material labeled "1" in Figure 5-c). Elements located below the free surface plane were modeled as isotropic elastic/plastic material with hardening dictated by the macroscopic uniaxial tension stress-strain data from Figure 1-c (material labeled "2" in Figure 5-c).

To approximate experimental loading conditions, for each model, displacement-controlled boundary conditions were applied to the two faces normal to the $x$ axis, simulating simple uniaxial tension. Displacements for nodes along the $x=0$ plane were fixed in the $\mathrm{x}$-direction, while nodes of the opposing face were displaced along the $x$ direction at a strain rate of $10^{-3} s^{-1}$, up to a total strain of $1.83 \%$. All other surfaces of the models remained traction-free. The stress-strain curves obtained on every subset (without the contribution of regions 1 and 2) are shown in Figure 5-d. The present approach of implementing boxes around the experimental subset lead to comparable stress-strain behavior to the experimental macroscopic curve (black dashed lines in Figures 5-d and 1-c). uniaxial displacement to all nodes on the top faces of the surface elements Simple uniaxial tension was applied to the resulting microstructure models, representative of the experimental loading conditions.

With this constitutive law, then for each material point, the following parameters were calculated: active slip, $\left(\dot{\gamma}^{(\alpha)}>0\right)$, accumulated slip, resolved shear stress for all slip systems, von Mises stress and reorientation (along with grain reference orientation deviation). 
a)

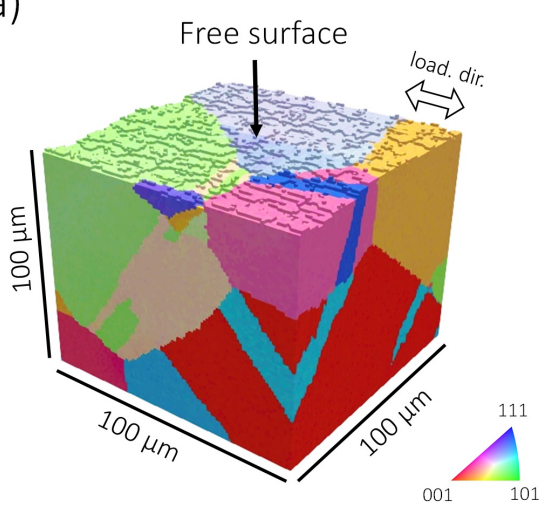

b)
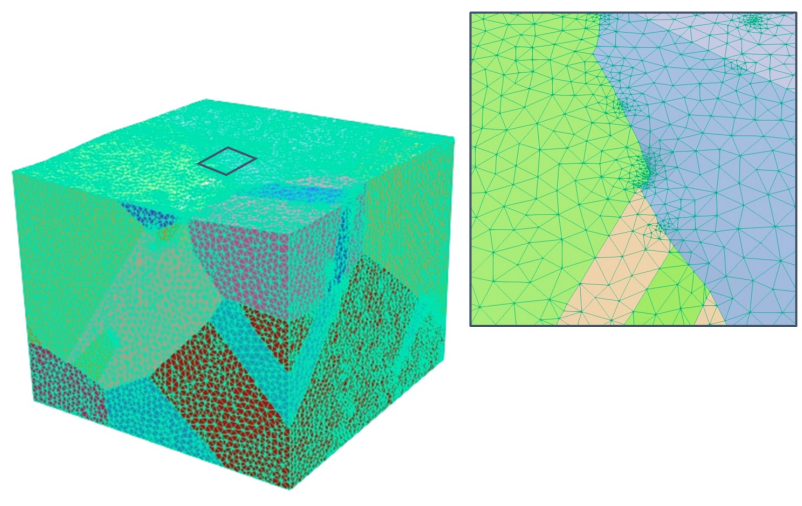

c)

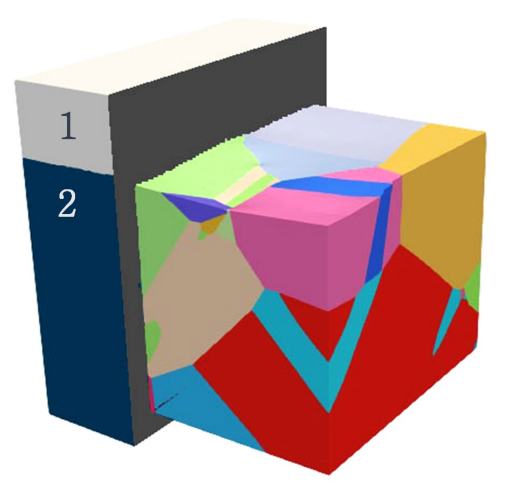

d)

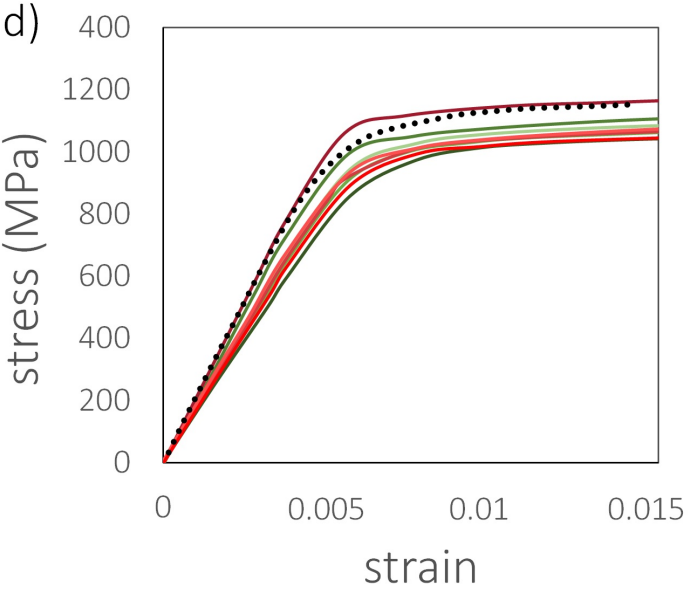

-tj w/ slip band 1

-tj w/ slip band 2

-tj w/ slip band 3

-tj w/ slip band 4

-tj w/ rotations 1

-tj w/ rotations 2

-tj w/ rotations 3

-tj w/ rotations 4

.• experimental curve

Figure 5: Crystal plasticity finite elements workflow: a) cropped experimental dataset in IPF colors according to the loading direction; b) meshed dataset, the zoomed-in area corresponds to the black square on top of the dataset; c) cropped dataset boxed with material of high compliance (label "1") on the free surface and isotropic material on other surfaces (label "2"); d) stress-strain responses for all investigated subset models.

\section{Results}

The merged dataset is presented in Figure 4-a. It contains 570 individual slip bands dispersed in a 3D microstructure containing 2546 grains (213 are visible on the free surface where HR-DIC measurements are performed), 12969 twin boundaries (433 emerge on the free surface) and 22440 triple junction lines (2902 are related to the grains on the free surface, including beneath the surface).

\subsection{Classification of slip band locations}

Visual inspection of the slip bands location reveals that they are often associated with three particular microstructural configurations. Examples of each are shown in Figure 6. The first one is denoted as parallel slip configuration [40]. This configuration involves a slip band that is located close and parallel to a coherent annealing twin boundary, 
as shown in Figure 6-a. In this example, two slip bands, shown in dark grey, have formed parallel and close to the twin boundaries highlighted with red dashed lines. This configuration has already been observed from DIC measurements in the same alloy $[12,13]$ and in other similar nickel base superalloys, such as Rene 88DT, Rene 65 and IN 100 $[19,41,42]$. These slip bands are known to form in the early stages of plasticity and are usually of high intensity [40]. They develop close to (but not on) the twin boundary planes, and run along the whole twin boundary length.

The second configuration involves triple junction lines and is referred to as triple junction configuration. Figure 6-b displays one example of this configuration, wherein a slip band is connected to the triple junction line formed by grains labelled Grain 1,2 and 3. This triple junction line is located in the bulk and emerges on the surface at the point marked by a red dot; while the slip band emerges at the free surface of the specimen to produce the trace highlighted with a white dashed line in Grain 1. The observation of the connection between the slip band and the triple junction line is only possible via the projection of the plane of the slip band into the 3D microstructure.

The last commonly observed configuration is related to the phenomenon of slip transmission, in which two slip bands lying in different grains are joined through their shared grain boundary. Three examples are shown in Figure 6-c, where each pair of slip bands is joined across a twin boundary in three neighboring grains, labeled Grain 4, 5 and 6. In these three particular cases, the intersection between the slip planes of the adjoining bands is a line that lies on the twin plane (highlighted with dashed red lines).

a) Parallel slip configuration

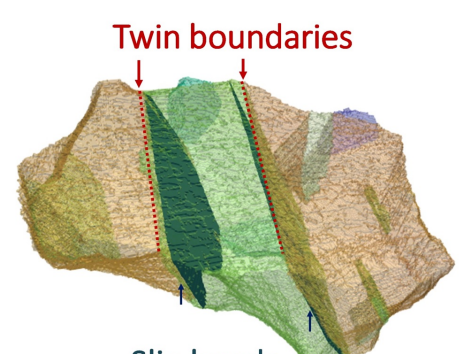

Slip bands b) Triple junction

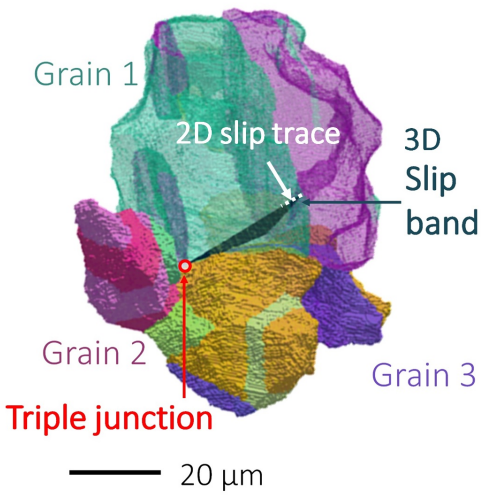

c) Slip transmission

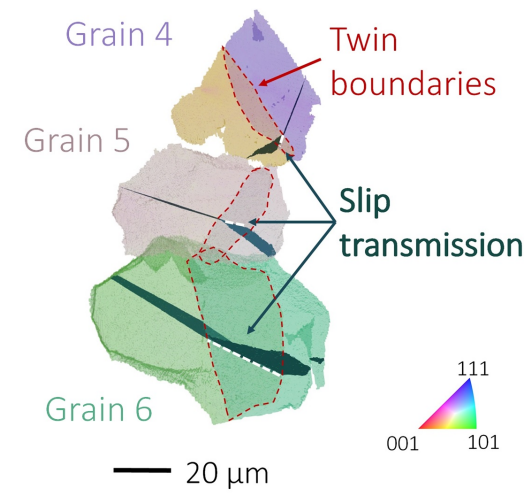

Figure 6: Three main types of slip band locations: a) parallel slip configuration in which slip bands develop parallel to and near annealing twin boundaries, b) slip band emanating from a triple junction line, c) slip transmission across grain boundaries.

\subsection{Statistics of slip bands configurations}

To determine relative amounts of each type of slip band, an automated procedure was developed in order to group all slip bands into one of these three configurations. To this end, a set of microstructure-based criteria was defined to automatically recognize the class of each band. A slip band with parallel slip character was identified based on the condition that it must be close and parallel to a twin boundary (using a threshold distance of $2 \mu \mathrm{m} / 2$ voxels). For the 
triple junction configuration, the connectivity of the slip bands voxels to triple junction lines voxels was considered. If both the slip band and triple junction line share at least seven voxels, the slip band is considered as emerging from the triple junction line. Finally, a pair of slip bands were considered to be in a transmission configuration if they belong to different crystals (including twin-related crystal orientations) and were connected to each other on the DIC surface within a distance of one voxel (which corresponds to the spatial accuracy of the multi-modal merging procedure and that of the 3D dataset). These three categories are not mutually exclusive. Among these, slip bands can fall into more than one. For instance, a slip band can be parallel to a twin boundary and connected to a triple junction line located below the surface. As other examples, it can be connected to a triple junction line but also transmitted to another slip band on the DIC surface or parallel to a twin boundary but in a transmission configuration. Consequently, seven categories of slip bands can be identified: parallel slip, parallel slip \& triple junction, triple junction, triple junction \& transmission, transmission, transmission \& parallel slip, and "other" configurations (i.e., bands that do not fit in any of the previous categories). 
a)
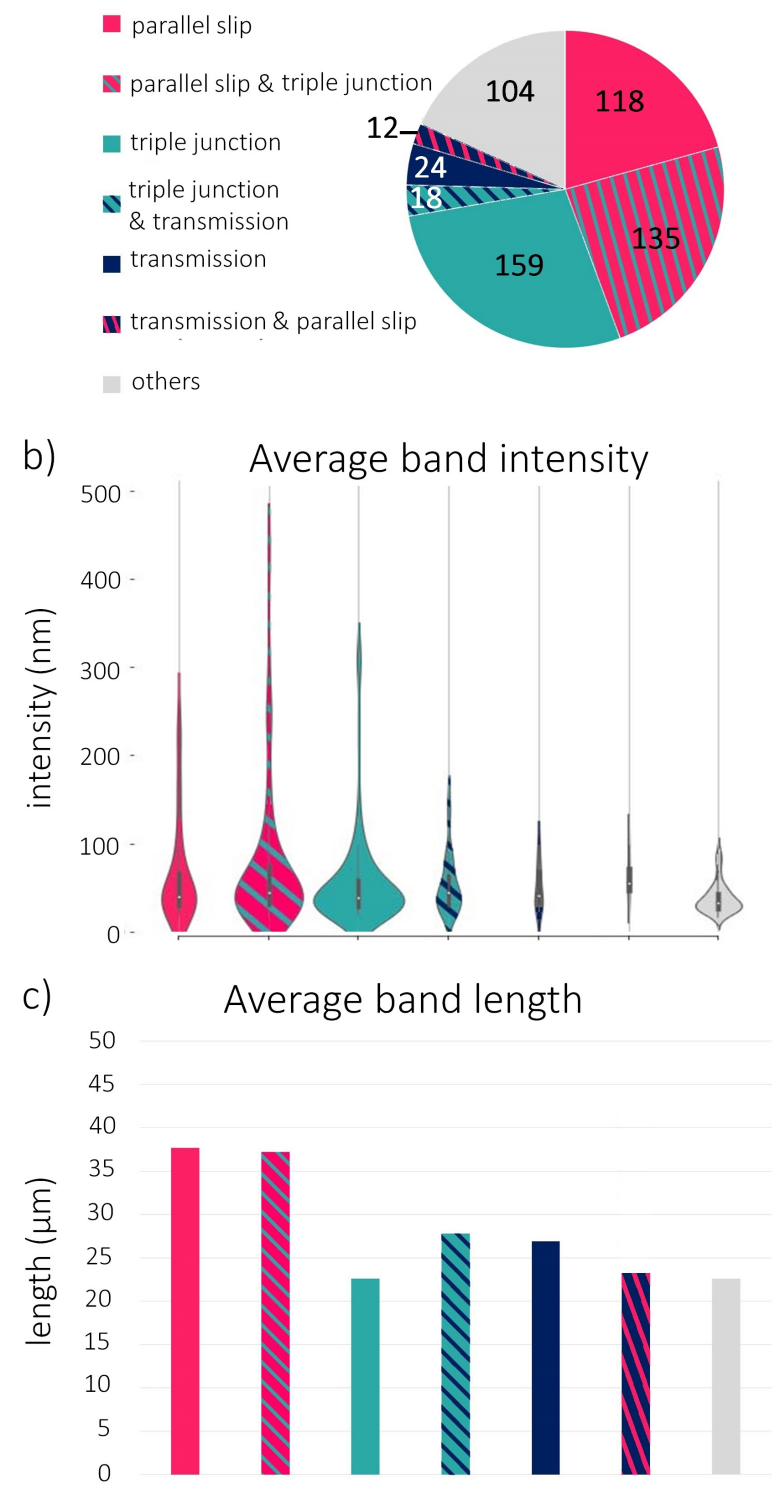

Figure 7: Statistical analysis of the different slip band types: a) number of slip bands, b) distribution of the bands average intensity, c) average band length.

Figure 7-a shows the relative amounts of slip bands in these seven classes. Over $80 \%$ of the slip bands can be assigned to at least one of the three configurations, indicating a strong correlation between slip bands and microstructure. It also indicates that these three configurations describe well the microstructural locations of a majority of the slip bands. Among these, the parallel slip and triple junction configurations dominate, constituting $96 \%$ of the slip bands that are in a determined configuration (pink and turquoise colors in figure 7, respectively). Similarly to numer- 
ous prior surface (2D) analyses [11, 12, 19], the most intense slip bands are often associated with twin boundaries, belonging to the parallel slip configuration group identified here. More strikingly, these statistics reveal that triple junction lines play at least an equally important role regarding slip band formation, being connected to $55 \%$ of them. The remaining 104 slip bands (gray area, 18\% of the slip bands), cannot be undeniably linked to a microstrucural feature. Examining these slip bands identified as others shows that most of them are embedded in small subgranular regions that developed significant lattice rotation during deformation, as shown in Figure 8.

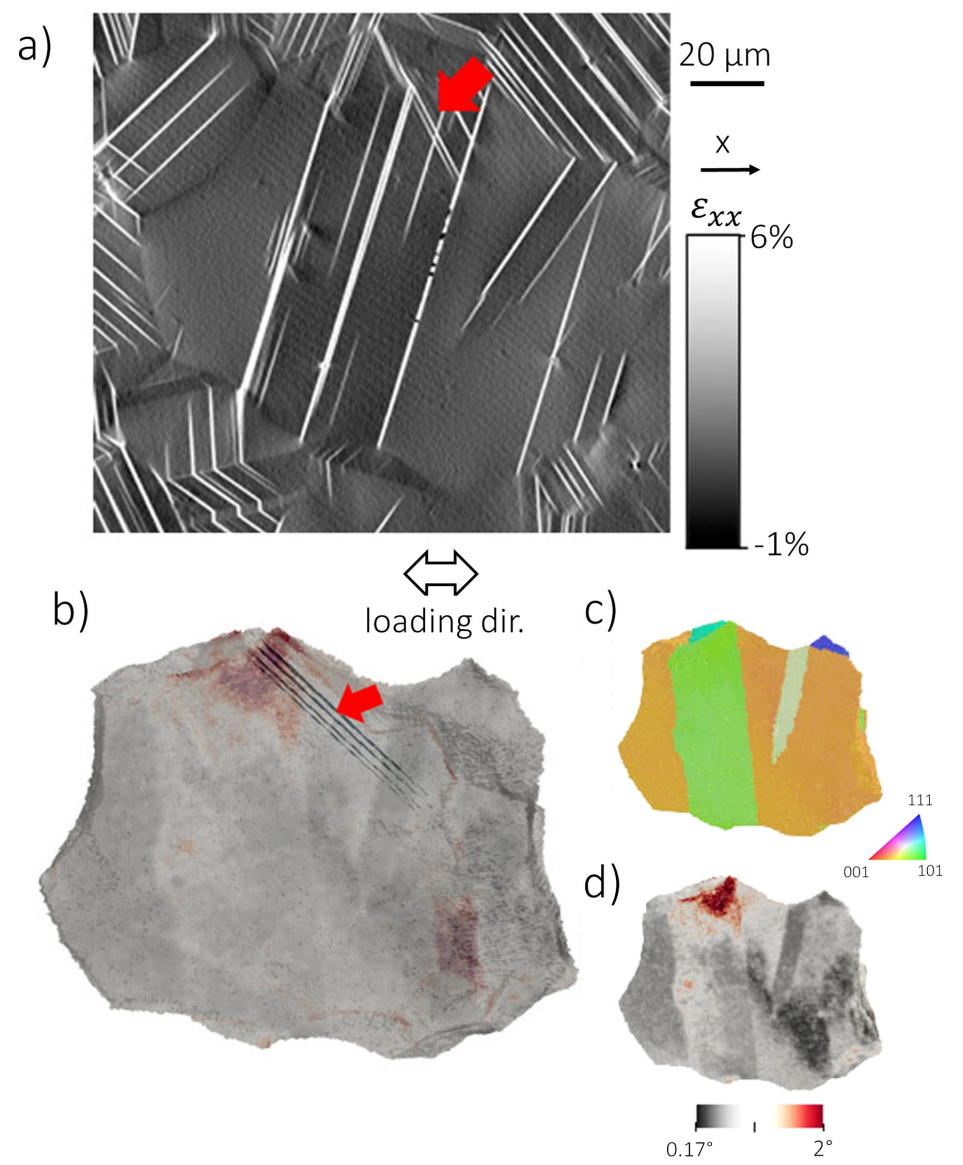

Figure 8: Correlation between regions of high lattice rotation and the location of slip bands: a) strain field $\epsilon_{x x}$ along the loading direction after deformation at $0.2 \%$ plastic strain b-d) corresponding 3D EBSD dataset and 3D representation of the slip bands within this particular grain; the color code in c) indicates the crystallographic orientation along the loading direction; the color code in b) and d) indicates the grain reference orientation deviation.

The length and intensity of every band were characterized, measured by the in-plane slip, to determine whether these properties are tied to the microstructure. Figures 7-b,c present the length and intensity of the bands in each of the seven categories. Slip bands in parallel slip and triple junction configurations are the longest and the most intense. In fact, the $10 \%$ most intense slip bands are in both parallel slip and triple junction configurations. This revealing 
result suggests that the combination of a twin boundary prone to parallel slip and triple junction line cause preferential localization of slip. The others category, however, consist entirely of low-intensity bands with no outliers or bands of outstandingly high intensity. They are also relatively short compared to all other slip bands in the same microstructure.

The strong correlations between the location of slip bands and regions of high lattice rotation to combinations of triple junction lines and twin planes enable identification of specific 3D grain structure environments as the main contributors to slip localization in the early stages of deformation. In particular, the significant role of triple junction lines on slip band formation is revealed here for the first time, and is made possible by the unique combination of HR-DIC and 3D EBSD measurements.

\subsection{Triple junction lines}

Based on 2D analyses, triple junctions have been identified as regions of high strain concentrations and preferential crack nucleation sites in various metallic systems [43-45]. As they involve three different crystals and due to their inherent 3D nature, triple junction lines are complex objects to describe analytically. In viewing them in 3D rather than 2D, they can be characterized by multiple geometric features, such as their length, global and local curvature, global or local dihedral angles between the grains; and crystallography related criteria, such as misorientations between crystals or differences in directional elastic modulus. These parameters likely affect the local mechanical fields around the triple junction lines, in comparison to the interior of the grains. A slip band and/or a zone of lattice rotation can develop in response to the magnitude and directionality of the complex stress or strain states generated at the triple junction line.

First, the relationships between the structure of the boundaries constituting the triple junction line and whether it is prone to form a slip band or lattice rotation are investigated. To identify junctions in the latter group, the average GROD around a triple junction line was calculated, considering a 5 voxel-thick zone selected around the junction line. Then only triple junction lines with average GROD greater than $1^{\circ}$ were considered as the ones that trigger significant lattice rotation. As a way of classifying their structure, a simple criterion proposed by Kobayashi et al. [43] was adopted for the classification of triple junction lines. This criterion considers the number of special and random boundaries they contain: a junction containing two twin boundaries and one random boundary is named R1; a triple junction line that contains two random boundaries is of type $\mathrm{R} 2$ and a junction made of three random boundaries is named R3. Figure 9 shows the number fraction of R1, R2 and R3 triple junction lines in grey bars for the first layer of grains in the experimental dataset. The distribution indicates that, unsurprisingly, R2 and R3-types are much more frequently observed than R1-types, which basically consist of the intersection of three twin boundaries. Interestingly, the number of R2 and R3 junctions are comparable due to the relative high density of annealing twins in the present material. In Figure 9, the green and teal colored distributions show the number fraction of triple junction lines that exhibit a slip band or high lattice rotation, respectively. Most of the junctions that are connected to a slip band are of R2-type, which means that they are composed by a twin boundary and two random boundaries. These junctions include the parallel slip $\mathcal{E}$ triple junction configuration presented in Figure 7 (section 5.2), but also triple junction 
lines which slip bands emanate from, that are not parallel to the twin boundary. An example of such a configuration is shown in Figure 11-a. In that particular dataset, a slip band emanates from a triple junction line that is made of the grains labelled 1, 2 and 3, where 3 is a thin twinned region within grain 2. This junction is of R2-type but the slip band is not in a parallel slip configuration. The triple junction lines that exhibit lattice rotation are mostly R3-type, in accordance with the findings from Kobayashi et al. [43]. These rotations likely arise from difficulty in accommodating deformation compatibility among the three crystals that form the junction line.

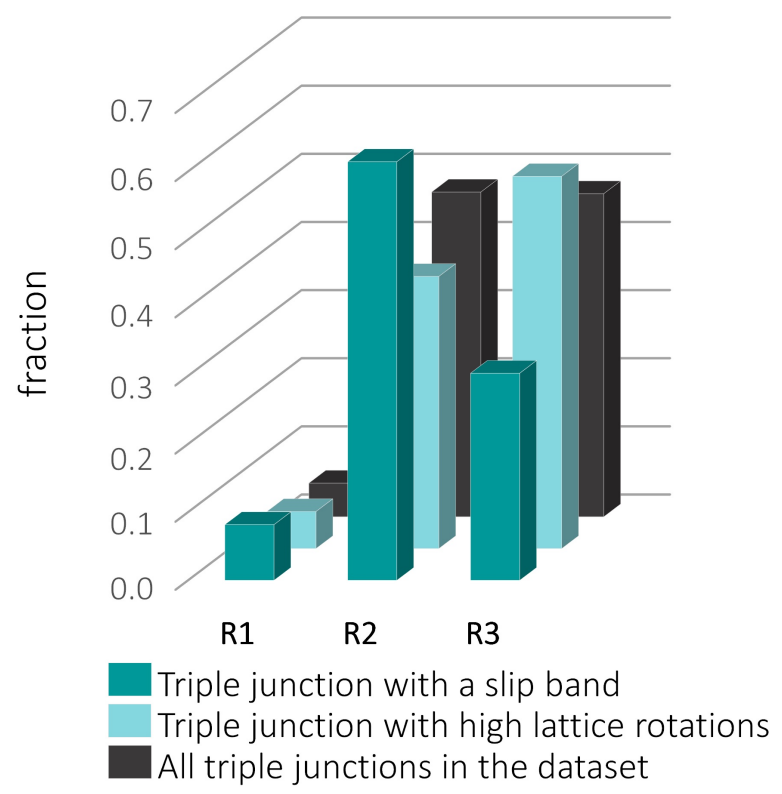

Figure 9: Number fraction distributions of triple junction lines within the first layer of grains according the number of random boundaries they contain (R1: one random grain boundary and 2 twin boundaries, R2: two random grain boundaries and one twin boundary, 3: three random grain boundaries.). The distributions corresponding to all triple junction lines, junction lines with high lattice rotation $\left(\mathrm{GROD}>1^{\circ}\right)$ and junction lines connected to a slip band are colored in dark grey, teal and green respectively.

While the triple junction lines connected to slip bands and/or high lattice rotation zones can be identified at a given strain, the underlying slip systems and the related mechanical fields and how they evolved with strain prior to the DIC measurements cannot. To gain insight into how these triple junction lines yielded to slip events, crystal plasticity simulations around some triple junction lines of interest were carried out.

\subsection{Crystal plasticity calculations around triple junction lines}

Several selected grain clusters and their triple junction lines were analyzed via the $\mathrm{CP}$ model to calculate the strain evolution in the lattice rotation and slip activity fields. Of particular interest is the propensity for each triple junction line to promote either lattice rotation or high intensity accumulated slip on a predominant slip system. Based on the statistical analyses and GROD mapping, several triple junction lines were identified, that were connected to either a 
zone of intense lattice rotation or an intense slip band. For each triple junction cluster, a reduced dataset measuring about $100 \mu \mathrm{m}$ on edge was extracted, meshed and analysed following the procedure described in section 4.2. The time-resolved slip activity, stress, and lattice rotation fields everywhere in the grain cluster (without the slip band) were calculated.

To enable comparisons of experimental and CPFE datasets, the simulation is performed in multiple strain steps that include the macroscopic strains at which HR-DIC measurements was acquired. While the HR-DIC dataset displays information at $0.2 \%$ plastic deformation, the 3D EBSD dataset was collected after straining at $1.26 \%$ plastic strain (1.83\% total deformation). Therefore, the CPFE simulation is performed to the final strain step, at $1.83 \%$ total deformation. The lattice rotation (GROD) maps from CPFE and 3D EBSD at 1.26\% plastic strain are compared in Figure 10, where a good agreement is observed. For all investigated datasets, very good agreement on both the intensity and distribution of the GROD is observed. 
a)

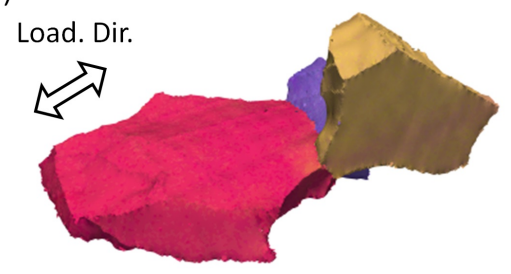

$20 \mu \mathrm{m}$

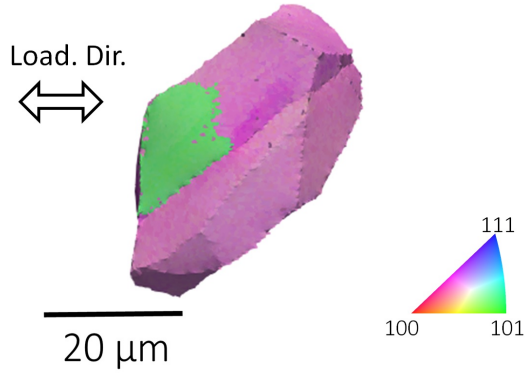

b)

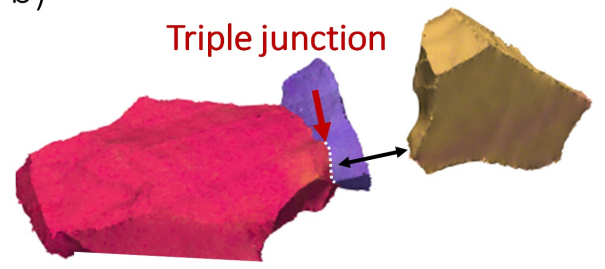

c)

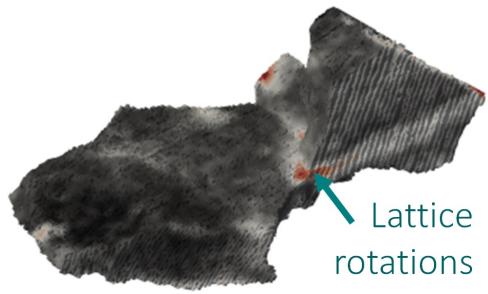

Experimental

d)
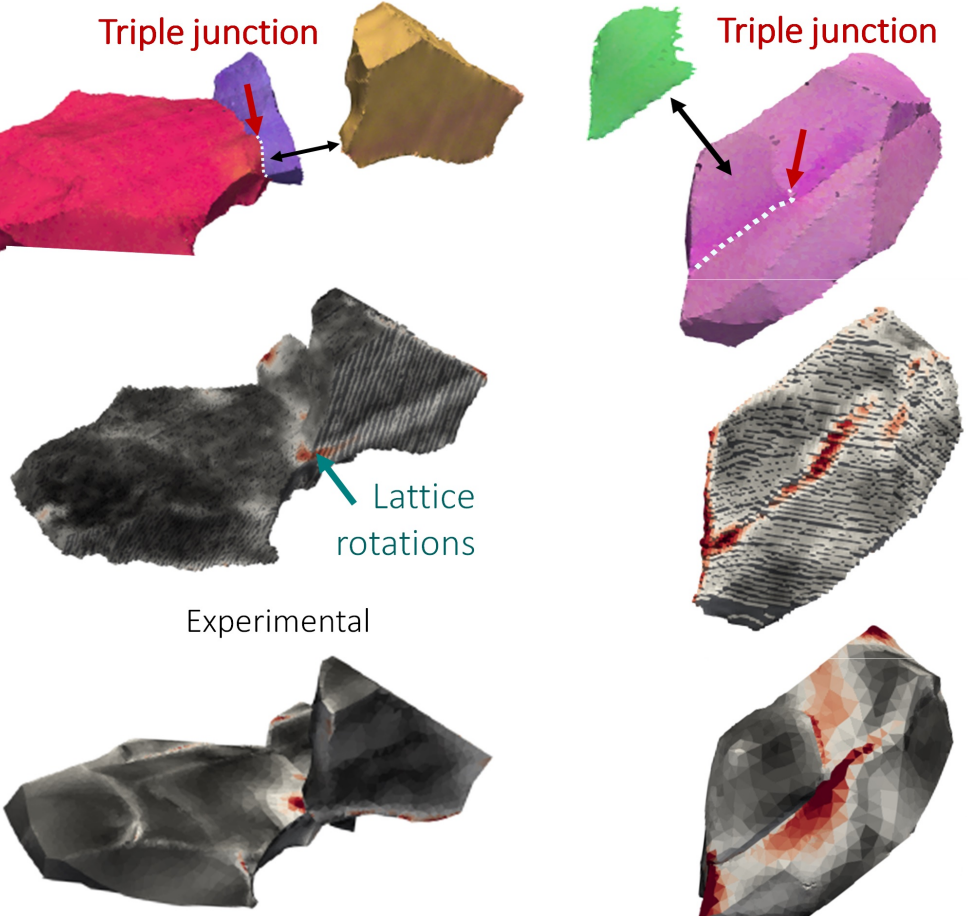

GROD $\left({ }^{\circ}\right)$

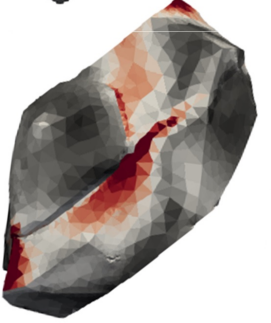

CPFE

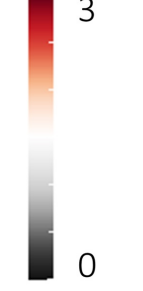

Figure 10: Comparison between the lattice rotation obtained experimentally and with CPFE in two triple junction lines: a) 3D view of the grains defining the triple junction lines of interest, in IPF colors according to the loading direction, b) split view showing the junction lines with white dashed lines, c) corresponding experimental GROD, d) CPFE GROD.

Figure 11 presents three grain clusters defining a triple junction line where an intense slip band is seen connected to it. In Figure 11-a, the orientation of each grain is mapped with respect to the loading direction. Using HR-DIC, the slip system in each slip band was determined, $\mathrm{C} 3$ in the first and A2 in the second and third (see Appendix 7.2 for the slip system nomenclature). Results from the CP calculations are shown in Figure 11-b to -d. In order to compare the relative activity in the vicinity of the triple junction line and the grain interior, a small region of interest (ROI) was selected around each triple junction line, shown in inserts in Figure 11-b. The activity around the triple junction line 
and the grain interior are depicted with plain and dashed lines, respectively. In Figure 11-b, the calculated evolution in accumulated slip in each system is shown. In the first case, slip accumulates preferentially on C3, both in the ROI and in the entire grain. Likewise in the next two cases, A2 is the most active slip system close to and on average over the entire grain. The evolution shows that in each case, one slip system is consistently preferred upon initial loading and over the entire course of straining. Figure 11-d shows that this slip activity is concomitant with relatively small intragranular lattice rotation. The GROD both near and far from the triple junction grows with strain, more or less homogeneously across the grain in which the slip band develops. Its value, however, remains relatively low $\left(<1^{\circ}\right)$. The rise in the von Mises equivalent stress in the ROI and over the entire grain are also provided in Figure 11-c. Unsurprisingly, the magnitude of the stress grows with strain and is higher in the ROI than over the entire grain. This occurs for all three triple junction line, in the experimental dataset.

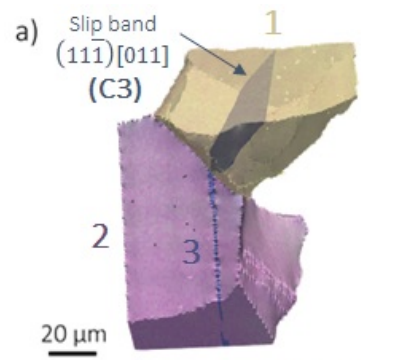

$\underline{20 \mu m}$

Slip band

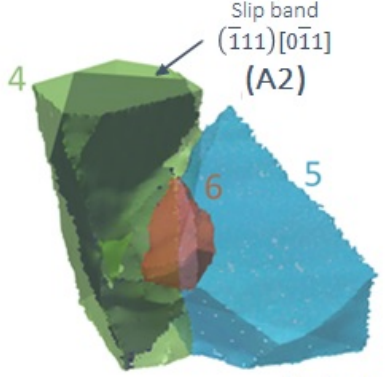

Slip band

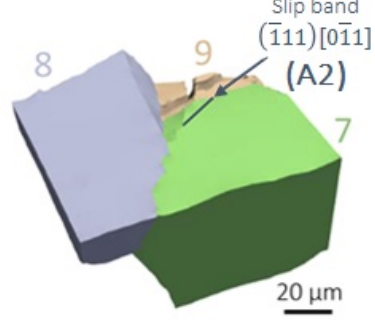

b) Mean cumulative slip
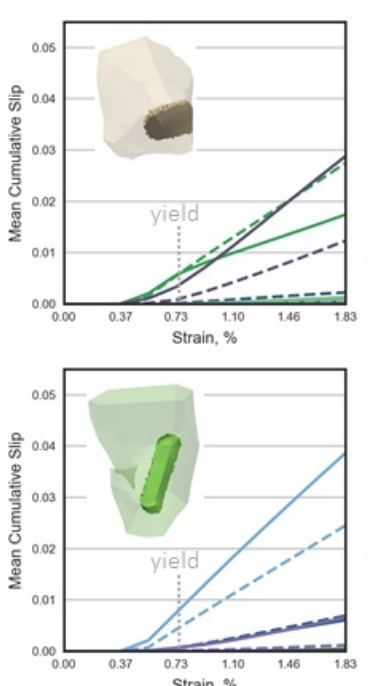

Strain, \%

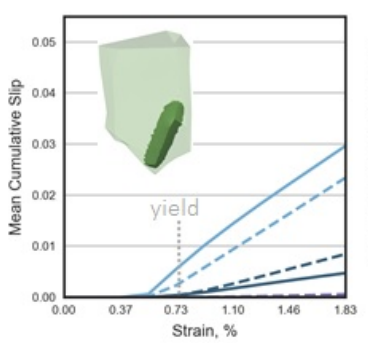

c) Mean von Mises stress
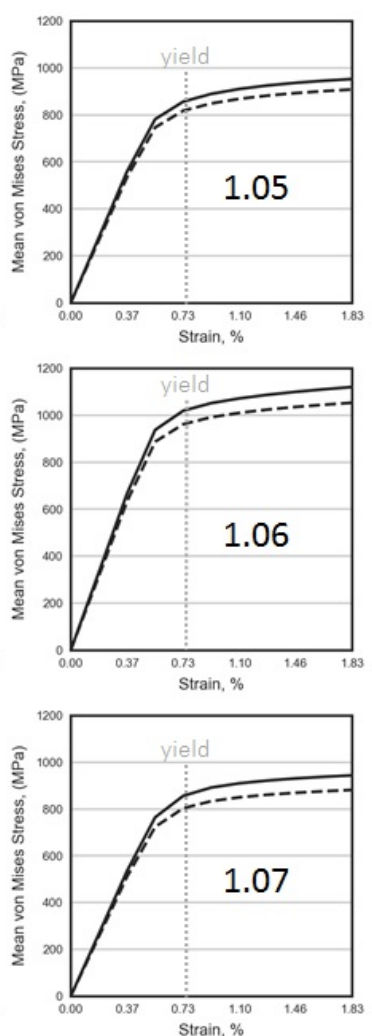

d)

Mean GROD
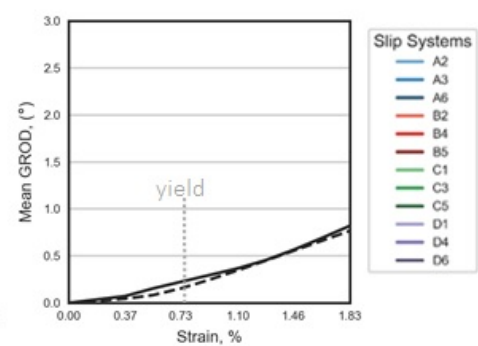

Figure 11: Plastic deformation in the reduced datasets showing a slip band connected to a triple junction, a) 3D view of the junctions of interest in IPF colors along the loading direction, b-d) CPFE calculations results in the regions of interest (ROI) highlighted in insert: b) mean cumulative slip on individual slip systems (identified by color), c) mean von Mises stress, d) mean GROD as a function of time (strain). In b-d, the activity around the ROI (close to the triple junction line) and on average in the grain is shown in solid and dashed lines, respectively.

On the other hand, two triple junction lines exhibiting lattice rotation but no slip bands were selected. Their grain clusters are shown in Figure 12 in IPF colors. The first triple junction is R3-type and the second one is R2-type, since 
its two largest grains are twin related. For these calculations, the ROI was picked within the grain(s) that exhibit the highest lattice rotation zones, which are grain 10 in the first cluster and grains 14 and 15 in the second. Figure 12-b presents the calculated strain evolution of accumulated slip. In no case is planar slip activity observed close to or far from the triple junction. Rather active slip on three distinct planes, signifying multi-slip condition, throughout straining. At least two slip systems are readily activated prior to yielding. Figure 12-c indicates that the value of the stress, measured by the von Mises stress, grows with strain and, in two of the three grains, is higher in the ROI than the entire grain. As seen for slip band-prone triple junction lines, no strong correlation between a high GROD and a stress concentration is found. It is worth noting that the slip bands of the two main systems should emerge at the surface if a slip band connected to the junction were to be triggered. Notably, the mean GROD close to the junctions is both high in all investigated grains $\left(>1^{\circ}\right)$ and significantly higher than the average GROD for the grain (dashed lines in figure 12-d). Thus, the multiple slip activated near the junction resulted in localized, large lattice rotation zones.

a)
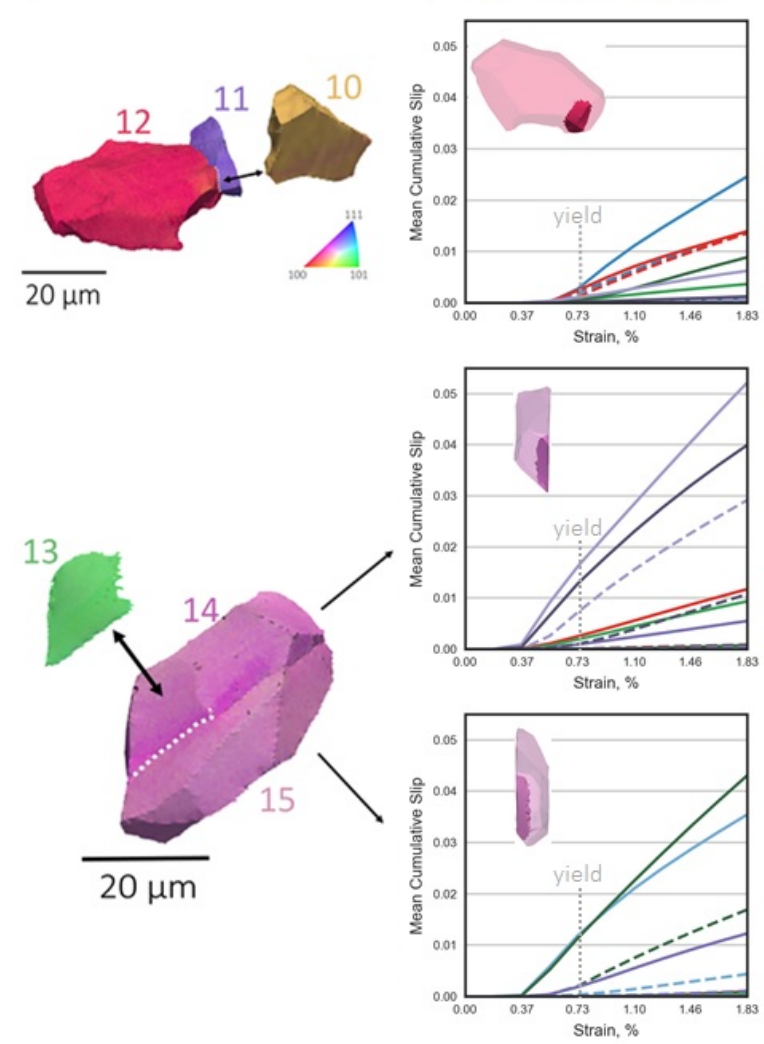

b) Mean cumulative slip

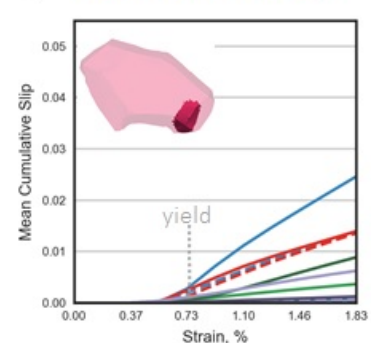

c) Mean von Mises stress
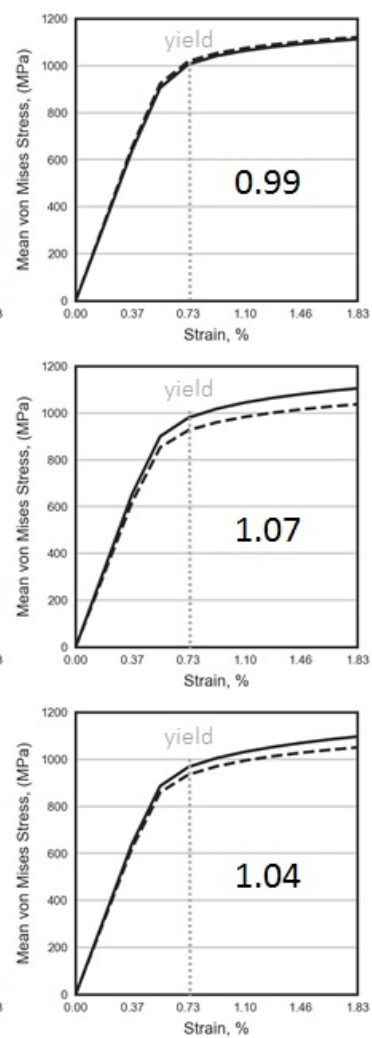

d) Mean GROD
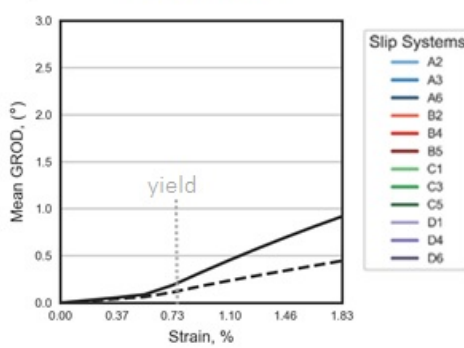

Figure 12: Plastic deformation in the reduced datasets showing high lattice rotation, a) 3D view of the junctions of interest in IPF colors along the loading direction, b-d) CPFE calculations results in the regions of interest (ROI) highlighted in insert: b) mean cumulative slip, c) mean von Mises stress, d) mean GROD as a function of time (strain). Macroscopic yielding corresponds to step 5. In b), the average cumulative slip over the whole grain that exhibits the slip band is shown in dashed lines. 


\section{Discussion}

Predicting failure in polycrystalline structures under conditions where extrinsic flaws are not responsible for early failure requires knowledge of the role of microstructural features in generating highly localized stresses and strains. While grain boundaries and triple junctions are known to develop significant stress or strain concentrations during loading, the specific character of the grain clusters that result in strain localization or highly concentrated lattice rotation is not understood. The investigation here has shown that the difficulty deformation compatibility among the joined grains in these particular configurations is accommodated by plastic deformation with specific signature of the form of lattice rotation and/or slip bands.

In this context, the present section discusses the possible roles of material properties, microstructural features, and ensuing slip processes responsible for the development of localized slip events in these particular regions. 3D statistical analyses presented in section 5.2 have demonstrated the importance of two particular microstructure configurations on incipient slip localization: the parallel slip configuration for slip bands parallel to twin boundaries and the triple junction configuration for slip bands emanating from triple junction lines. Both configurations are discussed in turn below.

\subsection{Twin boundaries triggering parallel slip}

The parallel slip configuration has been previously investigated in 2D for various metals and was observed to be the configuration yielding the strongest slip localization events during early plastic deformation $[9,40,46]$ and preferential sites for fatigue crack nucleation [3, 47-49]. Representative models and experimental datasets demonstrate the effect of the microstructural parameters of the parallel slip configuration on the intensity of slip developed near and parallel to annealing twin boundaries. The crystallographic orientation of the twin related crystals along the loading direction is of most importance according to $2 \mathrm{D}$ analyses [3, 9, 12, 40, 46-48]. Intense slip localization was reported either when the $\{111\}$ planes parallel to the twin boundary possess a high Schmid factor in either of the slip directions; either when the twin boundary separates crystal orientations that exhibit very large directional elastic modulus difference (in the loading direction). This is also observed in the present material. Figure 13-a shows the difference in elastic modulus across the twin plane, as a function of the Schmid factor of the active slip system in the slip band. Each marker corresponds to an individual slip band in parallel slip configuration. The markers are sized according to the length of the slip band and colored according to the intensity of in-plane slip (in $\mathrm{nm}$ ). The grey dashed line represents the maximum bounds of possible elastic modulus difference/Schmid factor combinations. In agreement with previous literature findings [12, 50], the most intense and longest slip bands are located along twin planes that exhibit either a high difference in elastic modulus but a medium Schmid factor, or a high Schmid factor and a lower difference in elastic modulus. These are respectively highlighted with circles " 1 " and " 2 " in the figure. The twin plane area is also reported to play a role, as it is similar to the area of the slip plane $[3,47]$. However, the intensity of the slip bands does not always correlate well with 2D measurements of twin boundary lengths [12]. This is believed to simply 
be the result of a cross-sectioning artifact, as a short twin boundary might be indicative of a small grain beneath the surface, or a grain that gets larger in the bulk and was cut on its outer part. In the present case, the 3D nature of the data allows for the extraction of the true twin plane area. The influence of the real twin plane area on the intensity of slip is shown in Figure 13-b: the longest and most intense slip bands are indeed located along the twin planes of largest area. Forty one of the 433 twin boundaries on the DIC surface favor parallel slip, which represents about 10\% of the total annealing twin boundaries.

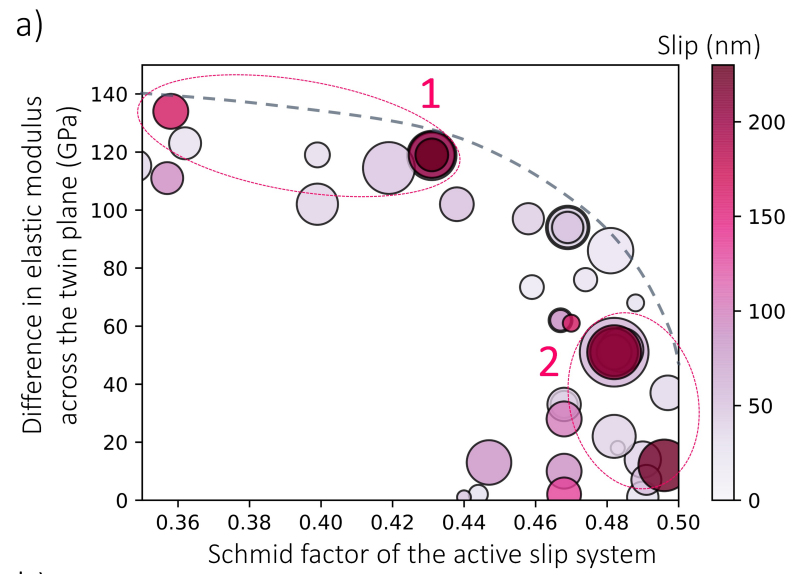

b)

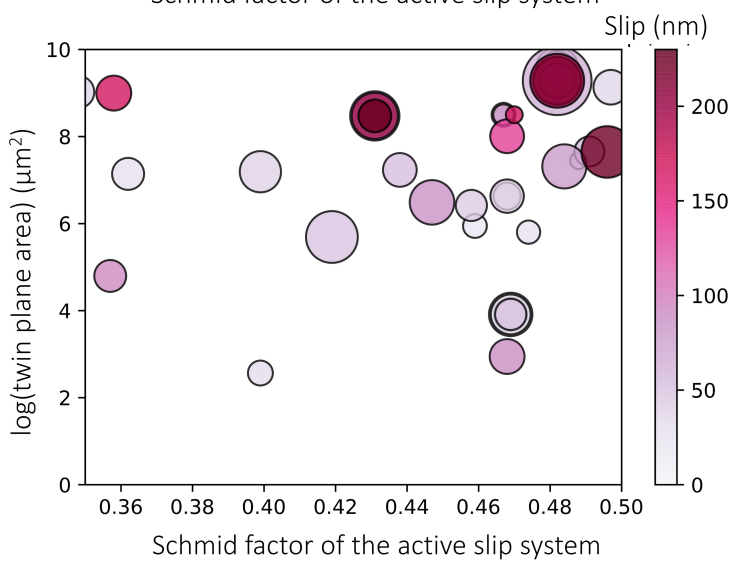

Figure 13: Characteristics of the twin boundaries triggering parallel slip: a) difference in directional elastic modulus across the twin plane (in the loading direction), b) twin boundary plane area (in log scale), both as a function of the Schmid factor of the active slip system (parallel to the twin plane). The size and color of the markers correspond to the length and the average intensity of in-plane slip within the band, respectively.

Remarkably, the slip bands of highest intensity are associated with both a twin boundary and a triple junction line. The discriminating role of the character of stress around triple junction lines is the focus of the next section.

\subsection{Local stress state near triple junction lines}

Taking into account the complete 3D nature of the microstructure has revealed, for the first time, the important contribution of triple junction lines on slip band formation. At macroscopic yield, more than half of the slip bands 
are connected to triple junctions within the bulk of the material. Mapping of local lattice rotation finds that triple junction lines can also induce high lattice rotation in their vicinity. These important features of triple junction lines are otherwise invisible when imaged from the free surface only. CPFE simulations of some of the grain clusters suggest that the outcome at a triple junction can be tied to the local slip activity generated there, specifically arising from a competition between multi-slip or single slip. Triple junction lines seen connected to a slip band, likely accommodated plastic deformation mostly by single slip virtually exclusively on one system, whereas those with high lattice rotation zones accommodate it with activation of multiple non-coplanar slip systems. Similar studies of triple junctions in pure aluminum tricrystal by Kashihara [51] found that the ratio between the primary slip system activity and secondary and tertiary slip systems activity is the main parameter to identify regions with high lattice rotation near triple junctions. What is not known about these slip bands in the present analysis is whether they are nascent bands or instead produced by a slip transfer event from a neighboring grain. One possible slip transfer event can be found in the triple junction shown in Fig. 11a with corresponding grain IDs 1, 2 and 3. There, the triple junction lies directly along a large area twin (grain 3), a well-known candidate for slip localization in the 718 alloy and thus possibly responsible for slip transfer into neighboring grain 1. It is likely these differing band origins produce characteristic features in local slip activity evolution, this presents a limitation of the classical CPFE model used in this study and a possible source of deviation with experimental results. Higher-order gradient crystal plasticity models with additional microscopic boundary conditions that account for slip transfer across grain boundaries could potentially shed light on these interactions [52-57]. The implication from the present slip activity calculations is that planar slip and high-lattice-rotation zones are two opposing states of slip activity. This insight would not have been realized from calculations of equivalent strain or von Mises stress measurements, which is commonly done. Indeed, no particular difference is observed in terms of ratio of von Mises stress around the junction line and the entire grains in either of Figures 11 and 10. The ratio was equal to 1 in both cases of emission of a slip band or the development of lattice rotation. This means that triple junction lines prone to slip banding or lattice rotation equally serve as stress concentrators (Figure 11-c). It was found slip activity is more discriminating than stress concentrations themselves.

While the CPFE simulations combined with slip localization and 3D microstructure measurements informed the importance of the slip systems activity in the vicinity of the triple junction lines, determination of the relevant microstructure parameters remains to be investigated. Figures 7 and 9 show that the presence of a twin boundary within the triple junction favors intense slip over lattice rotation. This is certainly related to easy activation of slip, parallel and near twin boundaries. However, the contribution of the orientation and morphology of the grains that make the junction, the 3D geometry of the junction itself, as well as the influence of the neighboring grains remain to be investigated. Understanding these effects is crucial for identifying the triple junction lines that are critical for slip localization and ultimately for fatigue crack nucleation. This is the object of future work. 


\section{Conclusions}

High resolution SEM DIC measurements were combined with 3D EBSD data using multi-modal merging methods, to create a rendering of slip bands in three dimensions over a cubic millimeter region of interest. Numerical tools were used to statistically investigate the location of hundreds of slip bands and have revealed strong correlations between the location of slip bands and the local configurations of annealing twin boundaries and triple junction lines. The $10 \%$ most intense slip events are related to twin boundaries that develop parallel slip and are simultaneously connected to triple junction lines. An analytical criterion involving the difference in directional elastic modulus across the twin plane, the Schmid factor and the twin plane area, allows for the identification of the twin boundaries that are likely to induce the formation of a slip band. CPFE calculations of the experimental 3D microstructure are carried out to predict time-resolved and spatially resolved intragranular slip activity and lattice rotation around triple junction lines. The model suggests that slip band formation occurs in grains associated with triple junction lines that produce predominantly planar slip on one main system under stress and that local lattice rotation is associated with a triple junction lines that locally cause multiple slip activity. These findings point to an important link between particular microstrutural features of triple junctions and the slip activity they generate, motivating development of specific microstruture-based criteria, a task for future work.

\section{Contributions}

MAC: 3D dataset acquisition, multi-modal data alignment and merging, post-processing and visualization scripts, statistical analyses, main manuscript contributor. JE: crystal plasticity calculations. JCS: project design, DIC dataset acquisition and post-processing, animations. ATP, MPE: 3D dataset acquisition. DT: transmission microscopy. IJB, TMP: funding and project design. All authors contributed to manuscript editing.

\section{Acknowledgements}

This work is funded by the U.S. Dept. of Energy, Office of Basic Energy Sciences Program DE-SC0018901. Use was made of computational facilities purchased with funds from the National Science Foundation (CNS-1725797) and administered by the Center for Scientific Computing (CSC). The CSC is supported by the California NanoSystems Institute and the Materials Research Science and Engineering Center (MRSEC; NSF DMR 1720256) at UC Santa Barbara. 


\section{References}

[1] J. Miao, T. M. Pollock, J. Wayne Jones, Crystallographic fatigue crack initiation in nickel-based superalloy René 88DT at elevated temperature, Acta Mater. 57 (20) (2009) 5964-5974, doi: \let \@tempa \bibinfo@X@doi10.1016/j.actamat.2009.08.022.

[2] J. Miao, T. M. Pollock, J. Wayne Jones, Microstructural extremes and the transition from fatigue crack initiation to small crack growth in a polycrystalline nickel-base superalloy, Acta Mater. 60 (6) (2012) 2840-2854,doi: \let \@tempa \bibinfo@X@doi10.1016/j.actamat.2012.01. 049.

[3] J. C. Stinville, E. Martin, M. Karadge, S. Ismonov, M. Soare, T. Hanlon, S. Sundaram, M. P. Echlin, P. G. Callahan, W. C. Lenthe, V. M. Miller, J. Miao, A. E. Wessman, R. Finlay, A. Loghin, J. Marte, T. M. Pollock, Fatigue deformation in a polycrystalline nickel base superalloy at intermediate and high temperature: Competing failure modes, Acta Mater. 152 (2018) 16-33, ISSN 13596454, doi: \let \@tempa \bibinfo@ X@doi10.1016/j.actamat.2018.03.035.

[4] H. Mughrabi, Cyclic Slip Irreversibilities and the Evolution of Fatigue Damage, Metallurgical and Materials Transactions A 40 (6) (2009) 1257-1279, ISSN 1543-1940, doi: \let\@tempa \bibinfo@X@doi10.1007/s11661-009-9839-8, URL http://dx.doi.org/10. $1007 /$ s11661-009-9839-8.

[5] H. Ho, M. Risbet, X. Feaugas, On the unified view of the contribution of plastic strain to cyclic crack initiation: Impact of the progressive transformation of shear bands to persistent slip bands, Acta Materialia 85 (2015) 155 - 167, ISSN 1359-6454, doi: \let\ @tempa\bibinfo@X@doihttp://doi.org/10.1016/j.actamat.2014.11.020, URL http://www.sciencedirect.com/science/article/ pii/S1359645414008647.

[6] D. Khireddine, M. Khireddine, Low cycle fatigue behaviour of an aluminium alloy with small shearable precipitates: Effect of surface coating, International Journal of Fatigue 22 (7) (2000) 585 - 591, ISSN 0142-1123, doi: \let \@tempa \bibinfo@X@doihttp://doi.org/10. 1016/S0142-1123(00)00028-1, URL http://www.sciencedirect.com/science/article/pii/S0142112300000281.

[7] Y. Brechet, F. Louchet, On the stability of orowan loops around coherent spherical ordered precipitates, Acta Metallurgica 37 (9) (1989) 2469 - 2473, ISSN 0001-6160, doi: \let\@tempa\bibinfo@X@doihttp://dx.doi.org/10.1016/0001-6160(89)90044-8, URL http: //www . sciencedirect.com/science/article/pii/0001616089900448.

[8] J. C. Stinville, P. G. Callahan, M. A. Charpagne, M. P. Echlin, V. Valle, T. M. Pollock, Direct measurements of slip irreversibility in a nickel-based superalloy using high resolution digital image correlation, Acta Mater. 186 (2020) 172-189, ISSN 13596454, doi: \let \@tempa \ bibinfo@X@doi10.1016/j.actamat.2019.12.009.

[9] R. Jiang, F. Pierron, S. Octaviani, P. Reed, Characterisation of strain localisation processes during fatigue crack initiation and early crack propagation by SEM-DIC in an advanced disc alloy, Materials Science and Engineering: A 699 (2017) 128 - 144, ISSN 0921-5093, doi: \let \@tempa \bibinfo@X@doihttp://dx.doi.org/10.1016/j.msea.2017.05.091, URL http://www.sciencedirect.com/ science/article/pii/S0921509317307074.

[10] H. Ho, M. Risbet, X. Feaugas, A cyclic slip irreversibility based model for fatigue crack initiation of nickel base alloys, International Journal of Fatigue 102 (2017) 1 - 8, ISSN 0142-1123, doi: \let \@tempa \bibinfo@X@doihttps://doi.org/10.1016/j.ijfatigue.2017.04.007, URL http://www.sciencedirect.com/science/article/pii/S0142112317301780.

[11] J. Stinville, M. Echlin, D. Texier, F. Bridier, P. Bocher, T. Pollock, Sub-Grain Scale Digital Image Correlation by Electron Microscopy for Polycrystalline Materials during Elastic and Plastic Deformation, Experimental Mechanics (2015) 1-20ISSN 0014-4851, doi: \let \@tempa\ bibinfo@X@doi10.1007/s11340-015-0083-4,URLhttp://dx.doi.org/10.1007/s11340-015-0083-4.

[12] M. Charpagne, J. Stinville, P. Callahan, D. Texier, Z. Chen, P. Villechaise, V. Valle, T. Pollock, Automated and quantitative analysis of plastic strain localization via multi-modal data recombination, Materials Characterization 163 (2020) 1-16.

[13] J. Liu, N. Vanderesse, J.-C. Stinville, T. Pollock, P. Bocher, D. Texier, In-plane and out-of-plane deformation at the sub-grain scale in polycrystalline materials assessed by confocal microscopy, Acta Materialia 169 (2019) 260-274, ISSN 1359-6454, doi: \let\ @tempa\bibinfo@X@doihttps://doi.org/10.1016/j.actamat.2019.03.001,URL https://www.sciencedirect.com/science/article/ $\mathrm{pii/S135964541930134X.}$

[14] G. Rao, M. Kumar, M. Srinivas, D. Sarma, Effect of standard heat treatment on the microstructure and mechanical properties of hot isostat- 
ically pressed superalloy inconel 718, Materials Science and Engineering: A 355 (1) (2003) 114-125, ISSN 0921-5093, doi:〈let\@tempa \ bibinfo@X@doihttps://doi.org/10.1016/S0921-5093(03)00079-0, URL https://www.sciencedirect.com/science/article/pii/ S0921509303000790.

[15] A. Kammers, S. Daly, Self-Assembled Nanoparticle Surface Patterning for Improved Digital Image Correlation in a Scanning Electron Microscope, Experimental Mechanics 53 (8) (2013) 1333-1341, ISSN 0014-4851, doi:〈let〉@tempa \bibinfo@X@ doi10.1007/s11340-013-9734-5, URL http://dx.doi.org/10.1007/s11340-013-9734-5.

[16] M. A. Sutton, Digital Image Correlation for Shape and Deformation Measurements, in: J. Sharpe, William N. (Ed.), Springer Handbook of Experimental Solid Mechanics, Springer US, ISBN 978-0-387-26883-5, 565-600, doi:〈let\@tempa \bibinfo@X@doi10.1007/ 978-0-387-30877-7_20, URL http://dx.doi .org/10.1007/978-0-387-30877-7_20, 2008.

[17] A. D. Kammers, S. Daly, Digital Image Correlation under Scanning Electron Microscopy: Methodology and Validation, Experimental Mechanics 53 (9) (2013) 1743-1761, doi: \let\@tempa \bibinfo@X@doi10.1007/s11340-013-9782-x, URL https://doi.org/10.1007/ s11340-013-9782-x.

[18] A. D. Kammers, S. Daly, Self-Assembled Nanoparticle Surface Patterning for Improved Digital Image Correlation in a Scanning Electron Microscope, Experimental Mechanics 53 (8) (2013) 1333-1341, doi:〈let @@tempa \bibinfo@X@doi10.1007/s11340-013-9734-5, URL https://doi.org/10.1007/s11340-013-9734-5.

[19] J. Stinville, M. Echlin, D. Texier, F. Bridier, P. Bocher, T. Pollock, Sub-Grain Scale Digital Image Correlation by Electron Microscopy for Polycrystalline Materials during Elastic and Plastic Deformation, Experimental Mechanics 56 (2) (2015) 197-216, doi:〈let\@tempa\ bibinfo@X@doi10.1007/s11340-015-0083-4,URLhttps ://doi.org/10.1007/s11340-015-0083-4.

[20] W. C. Lenthe, J. C. Stinville, M. P. Echlin, Z. Chen, S. Daly, T. M. Pollock, Advanced detector signal acquisition and electron beam scanning for high resolution SEM imaging, Ultramicroscopy 195 (2018) 93-100, doi:〈let @ tempa \bibinfo@X@doi10.1016/j.ultramic.2018.08.025, URL https://doi.org/10.1016/j.ultramic.2018.08.025.

[21] V. Valle, S. Hedan, P. Cosenza, A. L. Fauchille, M. Berdjane, Digital Image Correlation Development for the Study of Materials Including Multiple Crossing Cracks, Experimental Mechanics 55 (2) (2014) 379-391, doi:〈let @ tempa|bibinfo@X@doi10.1007/s11340-014-9948-1, URL https://doi.org/10.1007/s11340-014-9948-1.

[22] F. Bourdin, J. Stinville, M. Echlin, P. Callahan, W. Lenthe, C. Torbet, D. Texier, F. Bridier, J. Cormier, P. Villechaise, T. Pollock, V. Valle, Measurements of plastic localization by heaviside-digital image correlation, Acta Materialia 157 (2018) 307-325, doi:〈let \@tempa \bibinfo@ X@doi10.1016/j.actamat.2018.07.013, URL https ://doi.org/10.1016/j.actamat.2018.07.013.

[23] M. P. Echlin, M. Straw, S. Randolph, J. Filevich, T. M. Pollock, The TriBeam system: Femtosecond laser ablation in situ SEM, Materials Characterization 100 (2015) 1-12, doi:〈let @ tempa\bibinfo@X@doi10.1016/j.matchar.2014.10.023, URL https://doi.org/10.1016/ j.matchar. 2014.10.023.

[24] M. P. Echlin, T. L. Burnett, A. T. Polonsky, T. M. Pollock, P. J. Withers, Serial sectioning in the SEM for three dimensional materials science, Current Opinion in Solid State and Materials Science 24 (2) (2020) 100817, doi:〈let \@tempa \bibinfo@X@doi10.1016/j.cossms. 2020.100817, URL https: //doi.org/10.1016/j.cossms. 2020.100817.

[25] Y. H. Chen, S. U. Park, D. Wei, G. Newstadt, M. A. Jackson, J. P. Simmons, M. De Graef, A. O. Hero, A Dictionary Approach to Electron Backscatter Diffraction Indexing, Microscopy and Microanalysis 21 (03) (2015) 739-752, ISSN 1431-9276.

[26] M. D. Graef, M. Jackson, Saransh, Wlenthe, J. Kleingers, S. Wright, Josephtessmer, EMsoft-org/EMsoft: Release 4.2 to synchronize with DI tutorial paper, URL https://zenodo.org/record/2581285, 2019.

[27] M. A. Jackson, E. Pascal, M. D. Graef, Dictionary Indexing of Electron Back-Scatter Diffraction Patterns: a Hands-On Tutorial, Integrating Materials and Manufacturing Innovation 8 (2) (2019) 226-246.

[28] M. A. Groeber, M. A. Jackson, DREAM.3D: A Digital Representation Environment for the Analysis of Microstructure in 3D, Integrating Materials and Manufacturing Innovation 3 (1) (2014) 56-72, doi: |let\@tempa \bibinfo@X@doi10.1186/2193-9772-3-5, URL https: // doi.org/10.1186/2193-9772-3-5.

[29] J. Ahrens, B. Geveci, C. Law, Paraview: An end-user tool for large data visualization, The visualization handbook 717. 
[30] M.-A. Charpagne, F. Strub, T. M. Pollock, Accurate reconstruction of EBSD datasets by a multimodal data approach using an evolutionary algorithm, Materials Characterization 150 (2019) 184 - 198, ISSN 1044-5803, doi:〈let\@tempa \bibinfo@X@doihttps://doi.org/10.1016/j. matchar.2019.01.033, URL http://www.sciencedirect.com/science/article/pii/S1044580318329073.

[31] S. Kalidindi, C. Bronkhorst, L. Anand, Crystallographic texture evolution in bulk deformation processing of FCC metals, Journal of the Mechanics and Physics of Solids 40 (3) (1992) 537-569, ISSN 0022-5096, doi: \let @ tempa \bibinfo@X@doi10.1016/0022-5096(92)80003-9, URL https://www.sciencedirect.com/science/article/pii/0022509692800039.

[32] R. J. Asaro, Crystal Plasticity, Journal of Applied Mechanics 50 (4b) (1983) 921-934, ISSN 0021-8936, doi:〈let \@tempa \bibinfo@X@ doi10.1115/1.3167205, URL https ://doi .org/10.1115/1.3167205.

[33] D. Peirce, R. Asaro, A. Needleman, Material rate dependence and localized deformation in crystalline solids, Acta Metallurgica 31 (12) (1983) 1951 - 1976, ISSN 0001-6160, doi:|let〉@tempa|bibinfo@X@doihttps://doi.org/10.1016/0001-6160(83)90014-7, URL http: // www.sciencedirect.com/science/article/pii/0001616083900147.

[34] P. Franciosi, M. Berveiller, A. Zaoui, Latent hardening in copper and aluminium single crystals, Acta Metallurgica 28 (3) (1980) 273 - 283, ISSN 0001-6160, doi: \let〉@tempa \bibinfo@X@doihttps://doi.org/10.1016/0001-6160(80)90162-5, URL http: //www.sciencedirect. com/science/article/pii/0001616080901625.

[35] H. Mecking, U. Kocks, Kinetics of flow and strain-hardening, Acta Metallurgica 29 (11) (1981) 1865-1875, ISSN 0001-6160, doi: \let〉 @tempa\bibinfo@X@doihttps://doi.org/10.1016/0001-6160(81)90112-7, URL http://www.sciencedirect.com/science/article/ pii/0001616081901127.

[36] C. Sandström, Voxel2Tet, URL https://github.com/CarlSandstrom/Voxel2Tet, 2016.

[37] Y. Hu, Q. Zhou, X. Gao, A. Jacobson, D. Zorin, D. Panozzo, Tetrahedral Meshing in the Wild, ACM Trans. Graph. 37 (4) (2018) 60:160:14, ISSN 0730-0301, doi:〈let\@tempa\bibinfo@X@doi10.1145/3197517.3201353, URL http://doi .acm.org/10.1145/3197517. 3201353.

[38] Y. Hu, Q. Zhou, X. Gao, A. Jacobson, D. Zorin, D. Panozzo, Tetrahedral Meshing in the Wild, ACM Trans. Graph. 37 (4), ISSN 0730-0301, doi:〈let\@tempa|bibinfo@X@doi10.1145/3197517.3201353,URLhttps://doi.org/10.1145/3197517.3201353.

[39] A. Jacobson, L. Kavan, O. Sorkine-Hornung, Robust Inside-Outside Segmentation Using Generalized Winding Numbers, ACM Trans. Graph. 32 (4), ISSN 0730-0301, doi:〈let\@tempa|bibinfo@X@doi10.1145/2461912.2461916, URL https://doi.org/10.1145/2461912. 2461916.

[40] J. Stinville, N. Vanderesse, F. Bridier, P. Bocher, T. Pollock, High resolution mapping of strain localization near twin boundaries in a nickelbased superalloy, Acta Materialia 98 (2015) 29-42, ISSN 1359-6454, doi: \let @@tempa|bibinfo@X@doihttps://doi.org/10.1016/j.actamat. 2015.07.016, URL https://www. sciencedirect.com/science/article/pii/S1359645415004784.

[41] M. A. Charpagne, J. C. Stinville, A. T. Polonsky, M. P. Echlin, S. P. Murray, Z. Chen, N. Bozzolo, J. Cormier, V. Valle, T. M. Pollock, Tuning Strain Localization in Polycrystalline Nickel-Based Superalloys by Thermomechanical Processing, in: S. Tin, M. Hardy, J. Clews, J. Cormier, Q. Feng, J. Marcin, C. O’Brien, A. Suzuki (Eds.), Superalloys 2020, Springer International Publishing, Cham, ISBN 978-3-030-51834-9, 471-481, 2020.

[42] J. C. Stinville, W. C. Lenthe, M. P. Echlin, P. G. Callahan, D. Texier, T. M. Pollock, Microstructural statistics for fatigue crack initiation in polycrystalline nickel-base superalloys, International Journal of Fracture ISSN 1573-2673, doi:〈let @ tempa|bibinfo@X@doi10.1007/ s10704-017-0241-z, URL https://doi.org/10.1007/s10704-017-0241-z.

[43] S. Kobayashi, T. Inomata, H. Kobayashi, S. Tsurekawa, T. Watanabe, Effects of grain boundary- and triple junction-character on intergranular fatigue crack nucleation in polycrystalline aluminum, J. Mater. Sci. 43 (11) (2008) 3792-3799, ISSN 00222461, doi:〈let \@tempa \bibinfo@ X@doi10.1007/s10853-007-2236-z, URL https ://link.springer.com/article/10.1007/s10853-007-2236-z.

[44] A. Clair, M. Foucault, O. Calonne, Y. Lacroute, L. Markey, M. Salazar, V. Vignal, E. Finot, Strain mapping near a triple junction in strained Ni-based alloy using EBSD and biaxial nanogauges, Acta Mater. 59 (8) (2011) 3116-3123, ISSN 13596454, doi:〈let \@tempa \bibinfo@X@ doi10.1016/j.actamat.2011.01.051.

[45] M. Henning, H. Vehoff, Local mechanical behavior and slip band formation within grains of thin sheets, Acta Mater. 53 (5) (2005) 1285-1292, 
ISSN 13596454, doi: \let \@tempa \bibinfo@X@doi10.1016/j.actamat.2004.10.052.

[46] C. A. Stein, A. Cerrone, T. Ozturk, S. Lee, P. Kenesei, H. Tucker, R. Pokharel, J. Lind, C. Hefferan, R. M. Suter, A. R. Ingraffea, A. D. Rollett, Fatigue crack initiation, slip localization and twin boundaries in a nickel-based superalloy, Current Opinion in Solid State and Materials Science 18 (4) (2014) 244 - 252, ISSN 1359-0286, doi: \let \@tempa \bibinfo@X@doihttp://dx.doi.org/10.1016/j.cossms.2014.06.001, URL http://www.sciencedirect.com/science/article/pii/S1359028614000370, slip Localization and Transfer in Deformation and Fatigue of Polycrystals.

[47] A. Heinz, P. Neumann, Crack initiation during high cycle fatigue of an austenitic steel, Acta Metallurgica et Materialia 38 (10) (1990) 1933 - 1940, ISSN 0956-7151, doi: \let \@tempa bibinfo@X@doihttp://dx.doi.org/10.1016/0956-7151(90)90305-Z, URL http://www . sciencedirect.com/science/article/pii/095671519090305Z.

[48] O. Glushko, D. Kiener, Initiation of fatigue damage in ultrafine grained metal films, Acta Materialia 206 (2021) 116599, ISSN 1359-6454, doi: \let \@tempa\bibinfo@X@doihttps://doi.org/10.1016/j.actamat.2020.116599, URL https://www.sciencedirect.com/ science/article/pii/S1359645420310363.

[49] P. Villechaise, L. Sabatier, J. C. Girard, On slip band features and crack initiation in fatigued 316L austenitic stainless steel: Part 1: Analysis by electron back-scattered diffraction and atomic force microscopy, Materials Science and Engineering A 323 (1-2) (2002) 377-385, ISSN 09215093,doi:\let \@tempa \bibinfo@X@doi10.1016/S0921-5093(01)01381-8.

[50] J. Stinville, W. Lenthe, J. Miao, T. Pollock, A combined grain scale elasticplastic criterion for identification of fatigue crack initiation sites in a twin containing polycrystalline nickel-base superalloy, Acta Materialia 103 (2016) 461-473, ISSN 1359-6454, doi:\let\ @tempa \bibinfo@X@doihttps://doi.org/10.1016/j.actamat.2015.09.050,URLhttps://www.sciencedirect.com/science/article/ $\mathrm{pii/S1359645415007399.}$

[51] K. Kashihara, The Relationship between Crystal Rotation Axis Orientation and Active Slip System in Pure Aluminum Tricrystal Deformed in Compression, Materials and Metallurgical Transactions A 49 (3) (2008) 419-423, doi: \let $\ @$ tempa \bibinfo@X@doi10.2320/matertrans. MBW200704.

[52] A. Marano, L. Glbart, S. Forest, Intragranular localization induced by softening crystal plasticity: Analysis of slip and kink bands localization modes from high resolution FFT-simulations results, Acta Materialia 175 (2019) 262-275, ISSN 1359-6454, doi:\let\ @ tempa bibinfo@X@doihttps://doi.org/10.1016/j.actamat.2019.06.010,URLhttps://www.sciencedirect.com/science/article/ $\mathrm{pii} / \mathrm{S} 1359645419303696$.

[53] A. Marano, L. Glbart, S. Forest, FFT-based simulations of slip and kink bands formation in 3D polycrystals: Influence of strain gradient crystal plasticity, Journal of the Mechanics and Physics of Solids 149 (2021) 104295, ISSN 0022-5096, doi: \let \@tempa \bibinfo@X@doihttps: //doi.org/10.1016/j.jmps.2021.104295, URL https ://www.sciencedirect.com/science/article/pii/S0022509621000041.

[54] M. E. Gurtin, A. Needleman, Boundary conditions in small-deformation, single-crystal plasticity that account for the Burgers vector, Journal of the Mechanics and Physics of Solids 53 (1) (2005) 1-31, ISSN 0022-5096, doi: \let \@tempa \bibinfo@X@doihttps://doi.org/10.1016/j. jmps.2004.06.006, URL https://www.sciencedirect.com/science/article/pii/S0022509604001218.

[55] M. E. Gurtin, A theory of grain boundaries that accounts automatically for grain misorientation and grain-boundary orientation, Journal of the Mechanics and Physics of Solids 56 (2) (2008) 640-662, ISSN 0022-5096, doi: \let \@tempa \bibinfo@X@doihttps://doi.org/10.1016/j. jmps.2007.05.002, URL https://www.sciencedirect.com/science/article/pii/S0022509607001019.

[56] P. van Beers, G. McShane, V. Kouznetsova, M. Geers, Grain boundary interface mechanics in strain gradient crystal plasticity, Journal of the Mechanics and Physics of Solids 61 (12) (2013) 2659-2679, ISSN 0022-5096, doi: \let \@tempa \bibinfo@X@doihttps://doi.org/10.1016/j. jmps.2013.08.011, URL https://www.sciencedirect.com/science/article/pii/S0022509613001646.

[57] D. Gottschalk, A. McBride, B. Reddy, A. Javili, P. Wriggers, C. Hirschberger, Computational and theoretical aspects of a grain-boundary model that accounts for grain misorientation and grain-boundary orientation, Computational Materials Science 111 (2016) 443-459, ISSN 0927-0256, doi:〈let \@tempa\bibinfo@X@doihttps://doi.org/10.1016/j.commatsci.2015.09.048, URL https://www.sciencedirect. com/science/article/pii/S0927025615006291.

[58] E. Schmid, W. Boas, Kristallplastizitt, Springer-Verlag Berlin Heidelberg GmbH, Berlin, 1 edn., ISBN 978-3-662-34261-9, 1935. 
[59] G. Martin, N. Ochoa, K. Saï, E. Hervé-Luanco, G. Cailletaud, A multiscale model for the elastoviscoplastic behavior of Directionally Solidified alloys: Application to FE structural computations, Int. J. Solids Struct. 51 (5) (2014) 1175-1187, ISSN 0020-7683, doi: \let @tempa|bibinfo@X@doi10.1016/J.IJSOLSTR.2013.12.013. 


\section{Appendix}

\subsection{Von Neumann voxel neighborhood}

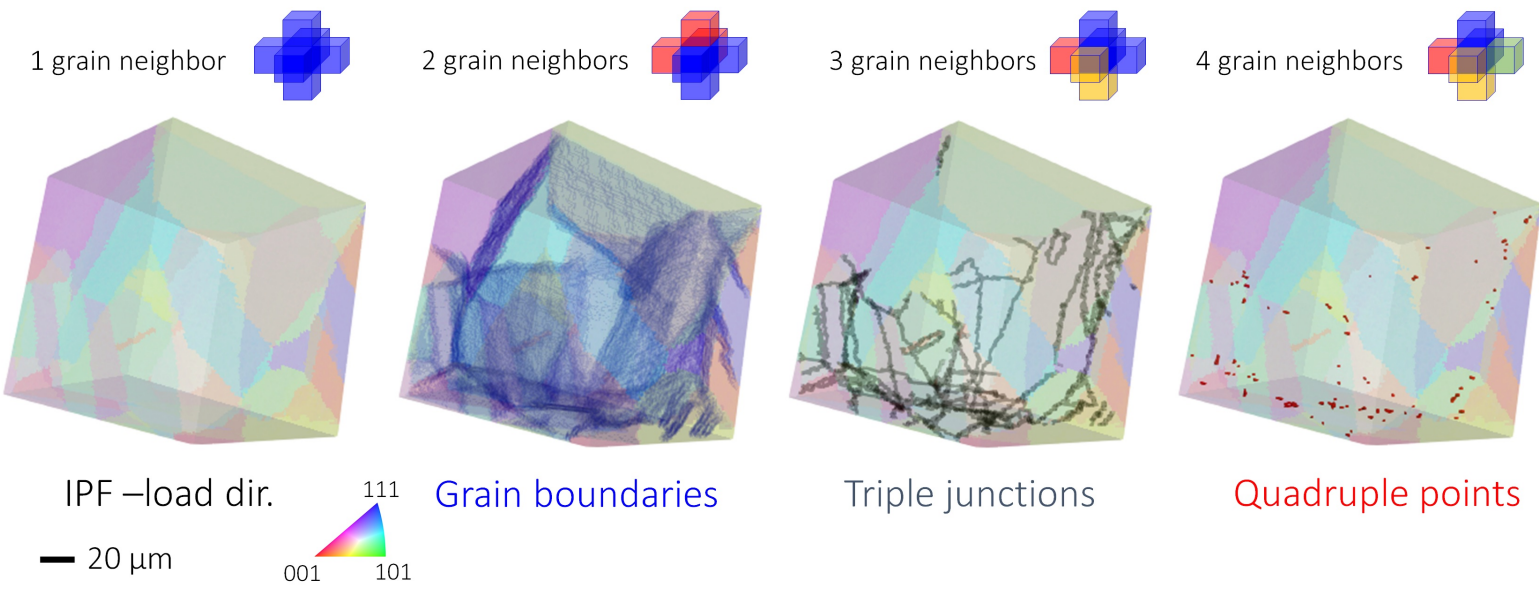

Figure 14: Illustration of the different voxel neighborhoods and corresponding microstructure.

\subsection{Slip systems nomenclature}

Table 2: $\{111\}\langle 110\rangle$ slip systems for FCC crystals with the notation of Schmid and Boas [58].

\begin{tabular}{ccc}
\hline Slip System & Slip Plane Normal & Slip Direction \\
\hline A2 & $(\overline{1} 11)$ & {$[0 \overline{1} 1]$} \\
A3 & $(\overline{1} 11)$ & {$[101]$} \\
A6 & $(\overline{1} 11)$ & {$[110]$} \\
B2 & $(111)$ & {$[\overline{0} 1]$} \\
B4 & $(111)$ & {$[\overline{1} 01]$} \\
B5 & $(111)$ & {$[\overline{1} 10]$} \\
C1 & $(\overline{11} 1)$ & {$[011]$} \\
C3 & $(\overline{1} 1)$ & {$[101]$} \\
C5 & $(\overline{11} 1)$ & {$[\overline{1} 10]$} \\
D1 & $(1 \overline{1} 1)$ & {$[011]$} \\
D4 & $(1 \overline{1} 1)$ & {$[\overline{1} 01]$} \\
D6 & $(1 \overline{1} 1)$ & {$[110]$} \\
\hline
\end{tabular}

\subsection{CPFE model parameter selection and calibration}

Values of elastic constants and initial dislocation density of IN718 were obtained from the literature [59]. Other material parameters used in the constitutive model were evaluated by fitting the simulated stressstrain curve of a 
representative volume element (RVE) to the experimental results. A 10x10x10 finite element RVE of the IN718 material was generated for calibration, consisting of 1000, eight-noded cubic mesh elements (C3D8), each element representing a single grain with a given crystallographic orientation sampled from a uniformly random orientation distribution. The RVE was tested with periodic boundary conditions in uniaxial tension. Figure 15 overlays the calculated stress-strain response of the RVE with the measured stress-strain data.

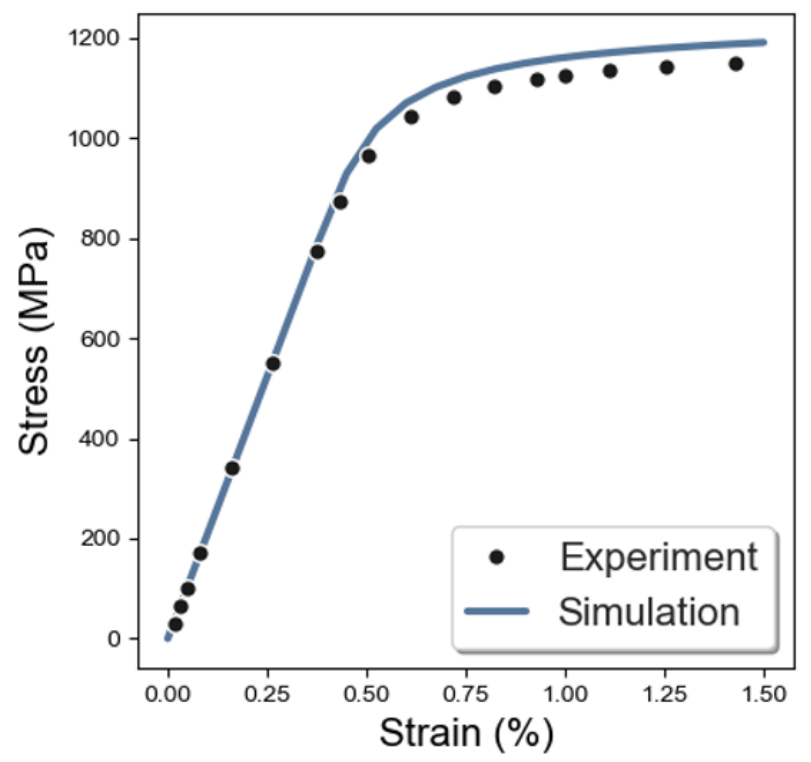

Figure 15: Engineering stress-strain curves measured by experiment and calculated with CPFE using fitted model parameters. 


\subsection{Smoothness of the mesh along triple junctions}

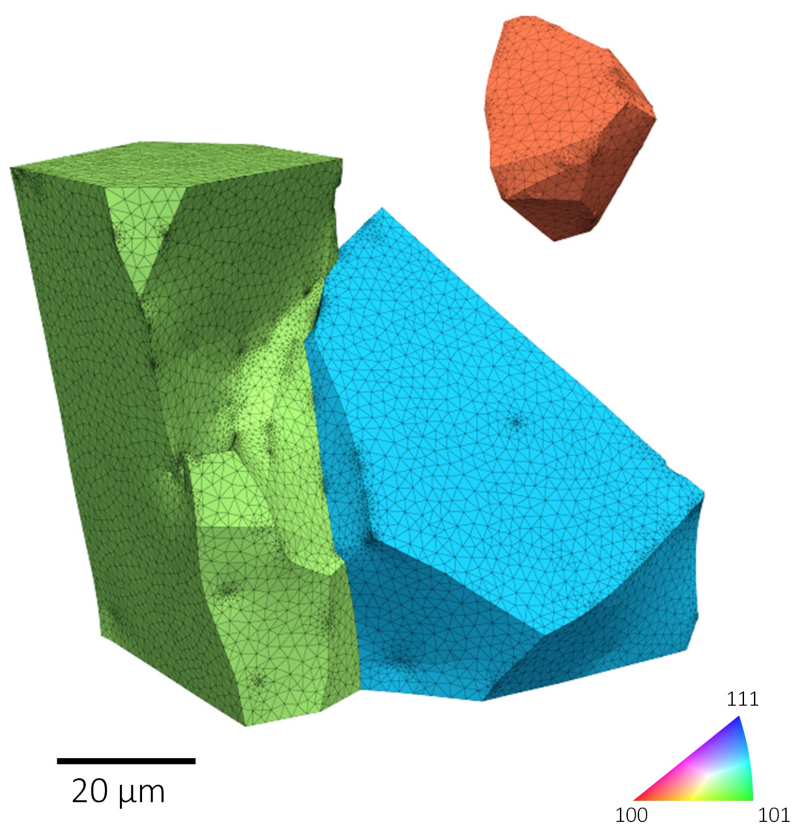

Figure 16: Example of meshing smoothness along a triple junction, the grains that make the junction are shown in IPF colors (loading direction). 